OPEN ACCESS

Edited by:

Karl Tsim,

Hong Kong University of Science and

Technology, Hong Kong

Reviewed by:

Ke Ma,

Shandong University of Traditional

Chinese Medicine, China

Shao Li,

Tsinghua University, China Xuetong Chen,

Northwest $A$ and F University, China

*Correspondence: Wuwen Feng

jiaoxiake-1@foxmail.com

Cheng Peng

pengchengcxy@126.com

Specialty section:

This article was submitted to

Ethnopharmacology,

a section of the journal

Frontiers in Pharmacology

Received: 25 August 2020 Accepted: 10 November 2020

Published: 19 January 2021

Citation:

Liu J, Feng $W$ and Peng C (2021) A Song of Ice and Fire: Cold and Hot

Properties of Traditional

Chinese Medicines.

Front. Pharmacol. 11:598744.

doi: 10.3389/fphar.2020.598744

\section{A Song of Ice and Fire: Cold and Hot Properties of Traditional Chinese Medicines}

\author{
Juan Liu ${ }^{1,2}$, Wuwen Feng ${ }^{1,2 *}$ and Cheng Peng ${ }^{1,2 *}$ \\ ${ }^{1}$ School of Pharmacy, Chengdu University of Traditional Chinese Medicine, Chengdu, China, ${ }^{2}$ State Key Laboratory of \\ Characteristic Chinese Medicine Resources in Southwestern China, Chengdu University of Traditional Chinese Medicine, \\ Chengdu, China
}

The theory of cold and hot properties is the basic theory of traditional Chinese medicines (TCMs) and has been successfully applied to combat human diseases for thousands of years. Although the theory of cold and hot is very important to guide the clinical application of TCMs, this ancient theory remains an enigma for a long time. In recent years, more and more researchers have tried to uncover this ancient theory with the help of modern techniques, and the cold and hot properties of a myriad of TCMs have been studied. However, there is no review of cold and hot properties. In this review, we first briefly introduced the basic theories about cold and hot properties, including how to distinguish between the cold and hot properties of TCMs and the classification and treatment of cold and hot syndromes. Then, focusing on the application of cold and hot properties, we take several important TCMs with cold or hot property as examples to summarize their traditional usage, phytochemistry, and pharmacology. In addition, the mechanisms of thermogenesis and antipyretic effect of these important TCMs, which are related to the cold and hot properties, were summarized. At the end of this review, the perspectives on research strategies and research directions of hot and cold properties were also offered.

\footnotetext{
Keywords: clinical application, antipyretic effect, thermogenesis, cold and hot properties, traditional Chinese medicines
}

\section{INTRODUCTION}

Traditional Chinese medicines (TCMs), an important category of complementary and alternative medicines, have been widely used in China and the surrounding areas for thousands of years (Zhang Q. et al., 2016). Because of the rich experience in combating various diseases and the increasing acceptance of complementary and alternative medicines, there is a growing trend to use TCMs as a therapeutic option to treat diseases in some Western countries including the United States, the United Kingdom, and Australia (Cheung, 2011; Lai et al., 2017; Porter et al., 2017; Chao et al., 2020). One of the characteristics of TCM is that the traditional Chinese culture and philosophy such as Yin and Yang are rooted in TCM (Huang et al., 2018), and thus TCM contains unique theories to guide the clinical application of TCMs. Those theories include cold and hot properties, five flavors, compatibility (combinational use of TCMs), and so on (Zhang J. H. et al., 2015; Wang Y. et al., 2016). For a TCM practitioner, he or she must obey those theories because violation of those theories would lead to the increase of toxicity or reduction of efficacy (Wang S. P. et al., 2012). For example, the combination of Gancao (the dried root and rhizome of Glycyrrhiza uralensis Fisch. ex DC.) and Gansui (the dried root of Euphorbia kansui S.L.Liou ex S.B.Ho) will cause side effects or even toxicity (Shen et al., 2016). 


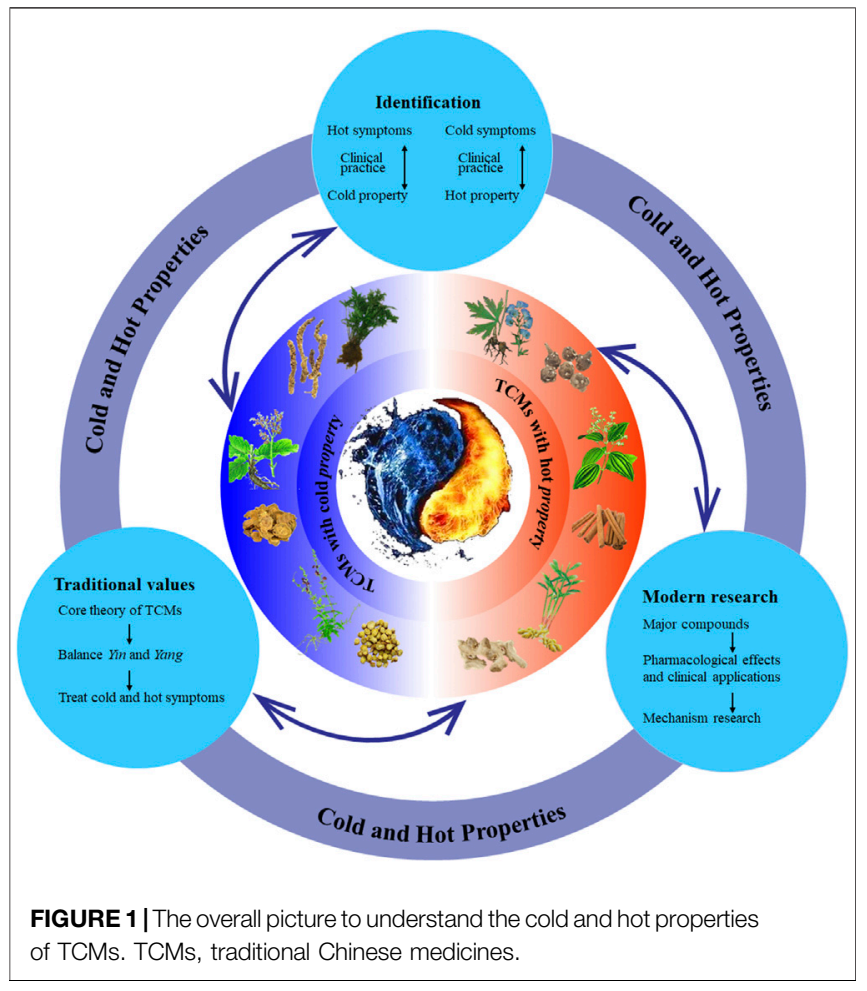

Among all the TCMs theories, the theory of cold and hot properties plays a fundamental role in the clinical practice of TCMs. This theory was first recorded in Shennong Bencao Jing (200-300 AD, Han Dynasty) and has been used by traditional Chinese practitioners to treat diseases for thousands of years. According to the rules of cold and hot properties recorded in Shennong Bencao Jing, patients with cold syndrome should be treated by TCMs with hot property and patients with hot syndrome should be treated by TCMs with cold property. The typical TCMs with cold property, like Zhimu (the dried rhizome of Anemarrhena asphodeloides Bunge) and Huanglian (the dried rhizome of Coptis chinensis Franch.), are used to relieve hot syndrome (Chen H. et al., 2015; Ota et al., 2019; Yu et al., 2020). And TCM practitioners applied TCMs with hot property, such as Fuzi (the processed lateral root of Aconitum carmichaelii Debeaux), to remedy cold syndrome (Rong et al., 2016). Even though thousands of years of clinical experience and modern pharmacological studies have proved the efficacy of those TCMs with strong cold or hot property, according to the book Yizong $B i d u$ (written by Li Zhongzi in the Ming dynasty), the violation of the application rules of cold and hot properties is not permitted because such a violation may lead to the failure of therapy and even aggravate the symptoms. For example, a coughing patient with hot syndrome has taken hot TCMs (the dried stem of Ephedra sinica Stapf, the twigs of Cinnamomum cassia (L.) J.Presl, the dried Zingiber officinale Roscoe, etc.) and finally exhibited dry mouth, nose and throat, persistent cough, excessive phlegm, and other hot syndromes, which is a typical case of hot TCMs being misused for hot syndrome resulting in hot syndrome aggravation (Zhang and Feng, 1987). Therefore, only when the medical practitioner has mastered the cold and hot properties, can the safety and effectiveness of TCMs be ensured.

As the core theory of TCM, cold and hot properties have been studied by more and more researchers due to its important role in guiding clinical diagnosis and treatment. With the development of new techniques, more and more efforts have been made in recent years to identify the cold and hot properties of TCMs, classify cold syndrome and hot syndrome, and study the effects of cold and hot TCMs on hot syndrome and cold syndrome. In addition, an increasing number of studies have focused on phytochemistry and pharmacology to investigate corresponding compounds associated with cold and hot TCMs and the mechanism of cold and hot TCMs. Although achievements have been made in the research of cold and hot properties, there has been no review of cold and hot properties. In this review, we summarized and discussed the studies of the cold and hot properties of TCM (Figure 1). We hope this review of hot and cold properties will help researchers to understand the art and science of cold and hot properties of TCM and finally to promote the rational use of TCM.

\section{THE IDENTIFICATION OF COLD AND HOT PROPERTIES OF TRADITIONAL CHINESE MEDICINES}

The identification of cold and hot properties mainly depends on the healthy human's direct feeling on a given TCM or the effects of TCMs on patients with cold or hot syndrome. For example, masticating mint leaves will produce a cold feeling, while chewing a piece of ginger will cause a hot feeling (Yu et al., 2020). Therefore, the mint leaf is classified as a cold TMC and ginger is classified as a hot TCM. When a person is ill, he or she may feel cold or hot and exhibit cold or hot syndrome. In general, the TCMs that can reduce or eliminate hot syndrome in a patient are cold in property. For example, Zhimu (the dried rhizome of Anemarrhena asphodeloides Bunge) and Zhizi (the dried ripe fruit of Gardenia jasminoides J.Ellis) are able to treat hot syndromes such as high fever, sweating, thirst, and powerful femtosecond pulse, so the properties of the two TCMs are cold (Chen, et al., 2005). On the contrary, the TCMs that can alleviate or eliminate cold syndrome are ordinarily hot in property. For instance, Fuzi (the processed lateral root of Aconitum carmichaelii Debeaux) and Rougui (the dried stem bark of Cinnamomum cassia (L.) J.Presl) are capable of treating chills, blood stasis, pain, stomachache, head, and pulse floating; thus, these two TCMs are hot in property (Zhou B. C. et al., 2019; Wang J. et al., 2020).

Although the theory of hot and cold properties is very important in clinical practice, this theory remains to be questioned because this theory mainly depends on human's sensory response and lacks scientific evidence. In recent decades, researchers have made a lot of efforts in animal models for studying the cold and hot properties of TCMs. These models for studying the cold and hot properties of TCMs can be divided into three types: animal models made with TCMs, biochemical or chemical drug-induced model, and cold exposure-induced model. For example, the cold syndrome 
induced by Huangbai (the dried stem bark of Phellodendron chinense C.K.Schneid.), Zhimu (the dried rhizome of Anemarrhena asphodeloides Bunge), etc. and the hot syndrome induced by Fuzi (the processed lateral root of Aconitum carmichaelii Debeaux), Rougui (the dried stem bark of Cinnamomum cassia (L.) J.Presl), etc. were used to study the properties of TCMs (Han X. Y. et al., 2018). Li et al. used collageninduced arthritis rat model to explore the effect of TCM formulas with cold and hot properties on the ultrastructures of synoviocytes (Li et al., 2002). In addition, cold-stress-induced hypothermia mice were used to study the effect of Fuzi (the processed lateral root of Aconitum carmichaelii Debeaux, a hot TCM) on hypothermia (Makino et al., 2009). With the development of new techniques, more and more efforts have been made in recent years to characterize the cold and hot properties of TCMs. Those methods include but not limited to microcalorimetry (Chen Z. et al., 2015), monitoring of animal thermotropism behavior (Zhao et al., 2011), cell temperature measurement (Yu et al., 2020), chemical space analysis (Fu et al., 2017), statistical pattern recognition (Wang X. Y. et al., 2012), bioinformatics analysis and chemical structure analysis (Liang et al., 2013), multisolvent similarity measurement (Wei et al., 2019), gene expression profile (Li A. R. et al., 2020), metabolomics, and network pharmacology ( $\mathrm{Li}$ et al., 2014; Xia et al., 2020). For example, based on the fact that the temperature of a living organism is directly related to energy production, Xiao et al. established a method for assessment of the hot properties of Fuzi (the processed lateral root of Aconitum carmichaelii Debeaux) according to the biothermodynamics parameters such as bacterial growth rate and energy release (Chen $\mathrm{Z}$. et al., 2015). At the animal level, Zhao et al. studied the cold and hot properties by observing the influence of TCMs on animal behaviors and functions, and it was found that cold TCMs enhanced thermotropism in animals and reduces energy metabolism, while hot TCMs had the opposite effects (Zhao et al., 2011). In short, animal model and modern technology have played important roles in the identification of cold and hot properties.

The cold and hot properties of TCMs mainly consist of four subtypes, including warm, hot, cold, and cool (Wang Y. et al., 2016). For a warm TCM, it is classified as a hot TCM, but its hot property is not strong. Similarly, for a cool TCM, it is classified as a cold TCM, but its cold property is not strong. It should be noticed that although the cold and hot properties of most TCMs can be easily identified, the cold and hot properties of some TCMs are not clear because they do not exhibit obvious cold and hot properties. And those TCMs are usually accepted as neutral TCMs. For example, TCMs such as Fuling (a fungus, the dried sclerotium of Poria cocos (Schw.) Wolf) and Dangshen (the dried root of Codonopsis pilosula (Franch.) Nannf.) are not classified as cold TCMs or hot TCMs, but neutral TCMs (Ung et al., 2007). Because neutral TCMs have no obvious effect on the clinical manifestations that are related to cold and hot syndromes, there are some challenges in the study of neutral TCMs, leading to few studies on neutral property. In this context, it is currently difficult to summarize the studies of neutral TCMs, especially the mechanism of neutral TCMs.
Therefore, this review mainly focuses on the hot and cold properties of TCMs.

\section{THE CLASSIFICATION AND TREATMENT OF COLD AND HOT SYNDROMES}

As a basic unit and the key concept in TCM theory, syndrome (ZHENG in Chinese) is a holistic summary of the patient's status and has been used to diagnose and treat disease for thousands of years (Li et al., 2007; Kanawong et al., 2012; Su et al., 2012). TCM practitioners distinguish between the conditions of individual patients according to the TCM syndrome. The cold syndrome and hot syndrome are the two most common and representative syndromes that represent two opposite but interrelated conditions of the human body (Zhou N. et al., 2019). Tongue appearance is a valuable diagnostic tool for determining syndrome in patients. According to the color and texture of the patient's tongue coating, the cold syndrome and hot syndrome are identified by a white-greasy and yellow-dense tongue coating, respectively (Jiang et al., 2012; Cui et al., 2019). In addition to tongue coating symptoms, cold syndrome and hot syndrome also show other symptoms. The cold syndrome also shows symptoms such as hypothermia, cold limbs, lost appetite, diarrhea, nausea, and vomiting (Mei, 2011). On the contrary, the hot syndrome generally has symptoms including high fever, unconsciousness, delirium, dysphoria, thirst, constipation, oral ulcer, sore in mouth, and dry eye (Mei, 2011; Zhang, 2011). According to this classification, TCMs with hot property are used to treat cold syndrome, whereas cold TCMs are used to treat hot syndrome (Zhou N. et al., 2019). For example, the representative cold TCMs, such as Huanglian (the dried rhizome of Coptis chinensis Franch.), Huangqin (the dried root of Scutellaria baicalensis Georgi), and Dahuang (the dried root and rhizome of Rheum palmatum L.), are extensively used for the treatment of hot syndrome (Xiao et al., 2019), whereas the typical hot TCMs, such as Fuzi (the processed lateral root of Aconitum carmichaelii Debeaux), Ganjiang (the dried Zingiber officinale Roscoe), and Rougui (the dried stem bark of Cinnamomum cassia (L.) J.Presl), are widely used to treat cold syndrome (Panthi et al., 2017). These cold TCMs and hot TCMs can be combined to form cold and hot formulas (Fu-Fang in Chinese) according to compatibility principle. These formulas in TCM also play a prominent role in the treatment of cold and hot syndromes. For example, one such formula for hot syndrome is the Gegen Qinlian Decoction, an ancient and effective treatment for dampness hot syndrome including diarrhea and dysentery, which originated from Shanghan Lun that is a canonical book of TCM compiled by Zhang Zhongjing (Li et al., 2014). And the classical TCM formula, Liuwei Dihuang Pills, with the function of nourishing kidney yin, is the major formula for Xiao Ke disease of yin deficiency and internal heat (Zheng et al., 2019). Moreover, Lily Bulb and Rehmannia Decoction has long been used as tonics for nourishing heart and lung, clearing heat and cooling blood in the treatment of mental instability, absent mindedness, dysphoria, and depression (Chi et al., 2019; Zhang et al., 2019; 


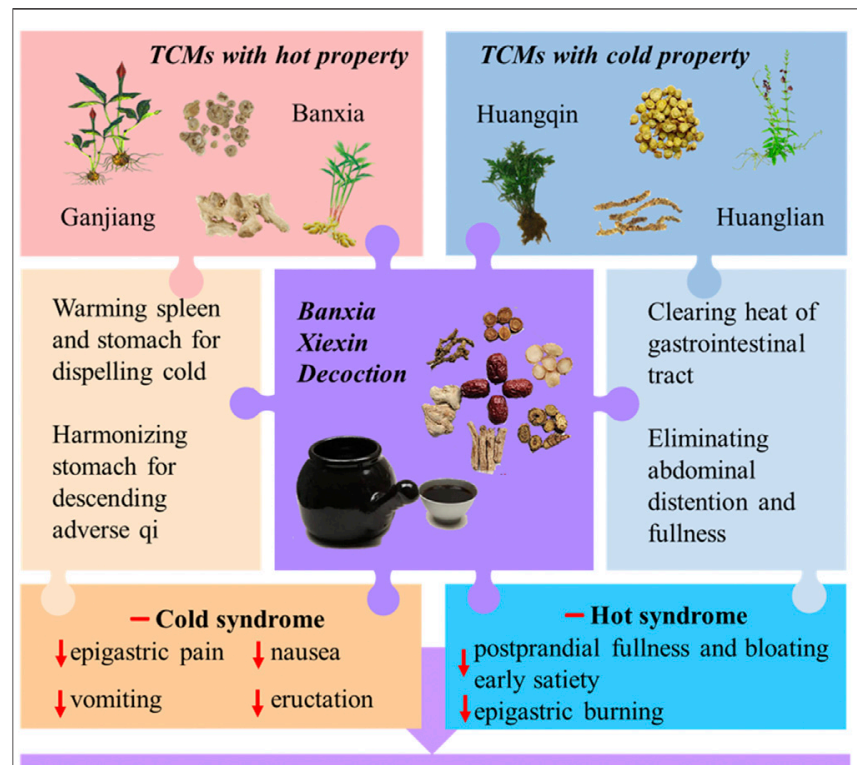

- Functional dyspepsia (FD) with cold and hot in complexity syndrome

FIGURE 2 | Banxia Xiexin Decoction for the treatment of functional dyspepsia with cold and hot complexity syndrome. TCMs, traditional Chinese medicines; $\downarrow$, decrease; -, inhibition.

Zhang et al., 2020). The above three examples are typical cases of TCM formulas for the treatment of hot syndrome. For cold syndrome, Sini Decoction, a classical TCM formula recorded in Shanghan Lun, has a significant therapeutic effect on cold symptoms displaying coldness on the extremities, vomiting, and diarrhea and is considered to be a therapy that has the essential effect of recuperating the patients from collapse (Cheng et al., 2016). Therefore, cold TCMs and hot TCMs, including single herb and formula, are indispensable for the treatment of cold and hot syndromes.

In addition to single cold syndrome or hot syndrome, patients often suffer a cold-hot complicated syndrome (Kemler et al., 2001). Patients with this syndrome can manifest cooling of limbs (representation of cold syndrome) and red tongue (representation of hot syndrome). Because the etiology and pathogenesis of cold-hot complicated syndrome are complicated, it is difficult to treat this syndrome by using cold TCMs or hot TCMs alone. For this complicated syndrome, formulas composed of cold and hot TCMs can be used (Chen et al., 2018). Shanghan Lun, a canonical book of TCM written by Zhang Zhongiing, contains a large number of examples of combinational use of cold and hot medicines, such as Banxia Xiexin Decoction, Xiao Chaihu Decoction (Shao and Li, 2019), and Wumei Pill (Yan et al., 2012). According to TCM theory, when formula composed of cold and hot TCMs is adopted, the hot syndrome can be treated by cold TCMs, and the cold syndrome can be treated by hot TCMs, eventually eliminating cold-hot complicated syndrome. Therefore, for cold-hot complicated syndrome, formulas composed of cold and hot TCMs are essential.
One such formula for cold-hot complicated syndrome is the Banxia Xiexin Decoction. It is an ancient and effective treatment for diarrhea (hot syndrome) and vomiting (cold syndrome) (Min, 2009; Zhao and Song, 2011). In the formula, Huanglian (the dried rhizome of Coptis chinensis Franch.) and Huangqin (the dried root of Scutellaria baicalensis Georgi) with cold property can clear heat and stop diarrhea; meanwhile, Banxia (the dried tuber of Pinellia ternata (Thunb.) Makino) and Ganjiang (the dried Zingiber officinale Roscoe) with hot property have the functions of eliminating cold and stopping vomiting. A randomized controlled trial involving patients from five centers evaluated the efficacy of modified Banxia Xiexin Decoction in the treatment of functional dyspepsia (FD) with cold-hot complicated syndrome. After 4 weeks of treatment, the results showed that, compared with the placebo group, the modified Banxia Xiexin Decoction significantly improved epigastric pain, postprandial fullness and bloating, early satiety, and a burning sensation in the stomach, indicating that it had a significant improvement effect on the FD with cold-hot complicated syndrome (Zhao et al., 2013a) (Figure 2). Another typical example involving cold-hot complicated syndrome is rheumatoid arthritis (RA). Many patients with RA show the hot symptoms including dry mouth, thirst, fever, and restlessness, as well as the cold symptoms including stiff joints, stabbing pain, sharp pain, and cold aggravated pain (Regestein, 2012). Guizhi-Shaoyao-Zhimu Decoction (GSZD), a formula composed of 9 TCMs such as Guizhi (the dried bark of Cinnamomum cassia (L.) J.Presl, hot in property) and Zhimu (the dried rhizome of Anemarrhena asphodeloides Bunge, cold in property) has been extensively used in the treatment of RA. Research has shown that GSZD can significantly improve cold syndromes (such as pain) and hot syndromes (such as redness and swelling) in RA (Guo et al., 2016). The above studies have further confirmed that the formulas composed of cold and hot TCMs have a clear therapeutic effect on cold-hot complicated syndrome. However, the mechanisms of these formulas in the treatment of cold-hot complicated syndrome is still unclear.

In recent years, there has been increasing concern about the application of bioinformatics and systems biology approaches to understand the development of diseases and the mechanism of the TCMs (Li and Zhang, 2013; Zheng et al., 2018; Tan et al., 2019; Wang X. et al., 2020). Based on the new trend in interdisciplinary fields, $\mathrm{Li}$ et al. established TCM systems bioinformatics (TCMSB) and proposed for the first time a map of "Phenotype network-Biological network-Herb network," which contributes to identifying network biomarkers for TCM syndrome and herb formula (Li et al., 2007; Li, 2009). In this context, the relationship between cold-hot complicated syndrome and formula could be transferred into a network context. For example, Li et al. (2007) investigated cold syndrome and hot syndrome in the context of the neuroendocrine-immune (NEI) system and identified the network biomarkers for both syndromes. They found that hormonerelated genes were predominant in the cold syndrome network, immune-related genes were predominant in the hot syndrome network, and neurotransmitter-related genes were distributed in both cold syndrome network and hot syndrome 
network. Therefore, TCM network pharmacology can be helpful to decipher the molecular mechanisms of TCM formulas for treating cold-hot complicated syndrome.

\section{THE IMPORTANT HOT AND COLD TRADITIONAL CHINESE MEDICINES AND THEIR SCIENTIFIC APPLICATION}

As the cold and hot properties of TCMs are regarded as the basic elements of the clinical practice of TCMs, their scientific applications have been in the spotlight from the researchers for a long time. Here, we took the major TCMs with hot property or cold property, namely, Fuzi (the processed lateral root of Aconitum carmichaelii Debeaux), Ganjiang (the dried Zingiber officinale Roscoe), Rougui (the dried stem bark of Cinnamomum cassia (L.) J.Presl), Huanglian (the dried rhizome of Coptis chinensis Franch.), Dahuang (the dried root and rhizome of Rheum palmatum L.), and Huangqin (the dried root of Scutellaria baicalensis Georgi) as examples to demonstrate the research progress on cold and hot properties of TCMs. These TCMs are taken as representatives for serval reasons. First, the cold property or hot property of these important TCMs is very clear according to the canonical books such as Shennong Bencao Jing. Secondly, these TCMs are frequently used in clinic and have prominent therapeutic effects on cold syndrome or heat syndrome. Thirdly, in the modern research of cold and hot properties of TCMs, these TCMs are often taken as typical research objects, and a large number of research achievements on the cold and hot properties of these TCMs have been accumulated.

\section{Important Hot Traditional Chinese Medicines for Cold Syndrome Fuzi}

Fuzi, the processed lateral root of Aconitum carmichaelii Debeaux (Ranunculaceae), is an herb extensively used in TCM to treat the diseases with cold symptoms because of its hot property, which was originally recorded in Shennong Bencao Jing (Zheng et al., 2014; Li C. et al., 2020). In Bencao Jingdu, it was viewed as the key TCM to ameliorate Yang deficiency and rescue the patient from prostration (Zhou et al., 2015). Interestingly, Huoshen Pai, a school of TCM, has become famous for the use of large doses of Fuzi to enhance Yang (Hong, 2012), which highlighted the importance of Fuzi in the treatment of cold syndrome.

Due to Fuzi's efficacy of reinforcing Yang, dispelling cold, and relieving pain, it was usually used to treat Yang depletion syndrome (a moribund syndrome with almost no appearance of Yang), Yang deficiency syndrome, and cold pain. Sini Decoction consisting of Fuzi, Ganjiang (the dried Zingiber officinale Roscoe), and Gancao (the dried root and rhizome of Glycyrrhiza uralensis Fisch. ex DC.), recorded in Shanghan Lun, is the most typical formula to illustrate the traditional usage of Fuzi. It can revive Yang and is suitable for cold symptoms such as prostration, cold sweat, and cold limbs, as well as weak and impalpable pulse. Shenfu Decoction composed of Fuzi and
Ginseng (the dried root and rhizome of Panax ginseng C.A.Mey.) is able to invigorate qi and restore Yang and is generally used to improve energy loss, cold extremities, sweating, plus feeble, and impalpable pulse, which originated from Shengji Zonglu. Sini Decoction and Shenfu Decoction are two representative formulas for the treatment of Yang depletion syndrome. Their application fully illustrates that Fuzi is the essential TCM to restore Yang and rescue the patient from collapse. In addition, Fuzi can also be applied to treat Yang deficiency syndrome (deficiency of spleen Yang, kidney Yang, and heart Yang) and cold-coagulation pain. For example, Fuzi Lizhong Pill plays an important role in warming the middlejiao and invigorating the spleen; thereby, it is favorably applied for patients with the deficient cold in spleen and stomach, cold pain in the abdomen, vomiting, diarrhea, and cold in limbs, which was recorded in Taiping Huimin Heji Jufang. For the deficiency of kidney Yang, Zhenwu Decoction in Shanghan Lun, composed of Fuzi, Fuling (a fungus, the dried sclerotium of Poria cocos (Schw.) Wolf; the dried sclerotium of Poria cocos (Schw.) Wolf), Baishao (the dried root of Paeonia lactiflora Pall.), Baizhu (the dried rhizome of Atractylodes macrocephala Koidz.), and so on, is an appropriate choice as it can warm and enhance kidney Yang to promote diuresis and improve swelling of the body. For the aspect of cold-coagulation pain, Gancao Fuzi Decoction, composed of Fuzi, Gancao (the dried root and rhizome of Glycyrrhiza uralensis Fisch. ex DC.), Baizhu (the dried rhizome of Atractylodes macrocephala Koidz.), and Guizhi (the dried bark of Cinnamomum cassia (L.) J.Presl), has cure function to wind-cold-dampness arthralgia syndrome. Taken together, these traditional usages of Fuzi further demonstrated its potent role in the treatment of cold syndrome, including Yang depletion syndrome (a moribund syndrome with almost no appearance of Yang), Yang deficiency syndrome, and cold pain.

Because Fuzi has a prominent effect on the treatment of cold syndrome, many modern studies have been carried out around it to explore the mystery of the traditional function of Fuzi. Phytochemical research has indicated that alkaloids such as aconitine, mesaconitine, hypaconitine, benzoylmesaconine, benzoylaconitine, and benzoylhypacoitine are the main bioactive constituents and the quality markers of Fuzi (Chen et al., 2008; Tang et al., 2012; Yu et al., 2015). In addition to alkaloids, a fraction of other ingredients has also been isolated and identified, including flavonoids, glucides, saponins, glycosides, fatty acids, ceramides, and uracil (Konno et al., 1985; Sang et al., 2005; Lyu et al., 2008; He et al., 2018; Sun et al., 2019; Zhao et al., 2020). The pharmacologic activities of Fuzi included, but were not limited to, thermogenesis (Deng et al., 2019); the effects on the cardiovascular system (Zhao et al., 2012), energy metabolism (Zheng et al., 2014), kidney protection (Tan et al., 2014), and immune system, anti-inflammation and analgesic action (Yang et al., 2020), antitumor (Zhang et al., 2017), hypoglycemic, and hypotensive effects, etc. (Huang et al., 2010). Based on the hot property and pharmacological effects of Fuzi, nowadays, it is used in clinical prescriptions for the treatment of shock, hypotension, coronary heart disease, chronic heart failure, diarrhea, syncope, neuralgia, joint pain, and rheumatoid arthritis (Zhou et al., 2015; Zuo et al., 2015). 
Since the thermogenesis of Fuzi is closely related to its hot property, the research of the mechanism of thermogenesis of Fuzi will help explain the hot property of Fuzi. From the perspective of body temperature, the water extract of Fuzi was founded to significantly restore the decrease in core body temperature in hypothermic mice induced by cold stress. Further research found that Fuzi can enhance the production of heat by increasing the expression of uncoupling protein 1 (UCP1), a protein that can mediate heat generation in brown fat tissue (BAT) (Makino et al., 2009; Harms and Seale, 2013). Specifically, peroxisome proliferator-activated receptors (PPARs) mediate the thermogenesis of Fuzi. The expressions of PPAR gamma $(\operatorname{PPAR}-\gamma)$ and PPAR coactivator 1-alpha (PGC- $1 \alpha)$ were upregulated by Fuzi treatment, and the activation of PPAR- $\gamma$ further stimulated the UCP1 expression (Makino et al., 2009). In addition, the hot property of Fuzi can be partially explained by its effect on energy metabolism. At the cellular level, Fuzi-containing serum could significantly increase $\mathrm{Na}^{+}-\mathrm{K}^{+}$-ATPase and $\mathrm{Ca}^{2+}{ }_{-}$ $\mathrm{Mg}^{2+}$-ATPase activities in hepatocytes, as well as intracellular ATP content, then promote cell energy metabolism, and increase intracellular energy reserves (Kan et al., 2018). Wang et al. found that the heat generation of rats could be increased by the water extract of Fuzi, and this process is associated with modulation of sugar metabolism, fatty acid metabolism, pentose phosphate pathway, and amino acid metabolism pathway (Wang et al., 2015). All these studies showed that the thermogenic mechanism of Fuzi involves the activation of BAT and the promotion of energy metabolism.

\section{Ganjiang}

Ganjiang (the dried Zingiber officinale Roscoe, or dried ginger in Chinese), another typical hot TCM, has been used as a food and herbal medicine in China, India, and other southeast Asian countries for thousands of years (Zhou et al., 2017). Ganjiang was first recorded in Mingyi Bielu in the Han Dynasty and it was described as a TCM with strong hot property (Chang et al., 1995; Fang et al., 2017; Zhou et al., 2017). Interestingly, Ganjiang can influence the hot property of Fuzi. A study based on animal thermotropism behavior found that, compared with the Fuzi group, the combination of Ganjiang and Fuzi significantly reduced the time and distance of mice moving on the warm plate, which indicated that Ganjiang enhanced the hot property of Fuzi (Sun et al., 2012).

In view of the efficacy of Ganjiang, it is widely used in prescriptions to treat patients with cold syndrome. Shanghan Lun and Jingui Yaolüe were respected as the ancestors of the prescriptions. Among them, numerous representative prescriptions containing Ganjiang are still widely used today for the treatment of cold syndrome. For example, the Sini Decoction in Shanghan Lun, a prescription composed of Ganjiang, Fuzi, and Gancao (the dried root of Glycyrrhiza uralensis Fisch. ex DC.), is one of the most classic formulas in a series of prescriptions containing Ganjiang. The combination of Ganjiang and Fuzi can not only increase the efficacy of Fuzi to restore Yang deficiency symptoms that manifested as prostration, deadly cold hand and foot, and weak pulse but also reduce the toxicity of Fuzi. For syndrome of deficient cold of spleen and stomach, Lizhong Pill in Shanghan Lun, a formulation composed of Ganjiang, Ginseng (the dried root and rhizome of Panax ginseng C.A.Mey.), and Baizhu (the dried rhizome of Atractylodes macrocephala Koidz.) has the function of invigorating spleen and qi. Thus, it can treat cold syndromes such as cold pain of epigastric abdomen, loss of appetite, reduced diet, vomiting, and diarrhea. Furthermore, Banxia Ganjiang San, a classical preparation recorded in Jingui Yaolüe and composed of Ganjiang and Banxia (the dried tuber of Pinellia ternata (Thunb.) Makino), has strong effects of dispelling cold and preventing vomiting. Because of the powerful effect of dispersing cold in the spleen and stomach, Ganjiang is regarded as a drug with broadspectrum antiemetic effect (Oh, 2003). The cold-dispelling function of Ganjiang can also relieve pain. Combinational use of Ganjiang and TCMs with hot property can enhance effect of Ganjiang to dispel cold and relieve pain. Erjiang Pill in Heji Ju Fang, consisting of Ganjiang and Gaoliangjiang (the dried rhizome of Alpinia officinarum Hance), exerts potent function of dispersing cold to improve pain in the heart and spleen. In addition, Ganjiang has the function of warming lung and can be used to treat cough and asthma caused by cold, cold in the back, and phlegm retention. For example, Xiaoqinglong Decoction consisting of Ganjiang, Xixin (the dried root and rhizome of Asarum heterotropoides F.Schmidt), Wuweizi (the dried ripe fruit of Schisandra chinensis (Turcz.) Baill.), Mahuang (the dried stems of Ephedra sinica Stapf), etc. is very effective for patients with fluid retention, cough, and asthma, which is registered in Shanghan Lun.

Ginger contains about 58\% oleoresin and 9\% lipids or glycolipids. The spicy taste of ginger is mainly affected by the $25 \%$ pungent components of oleoresin that is mainly composed of gingerol. Ginger contains up to $3 \%$ volatile oils, accounting for 20\%-25\% of oleoresin (Chrubasik et al., 2005). More than 200 compounds have been identified from ginger, and its biologically active components include volatile oils, anthocyanins, tannins, and spicy compounds (gingerol, sesquiterpenes, and shogaols) (Semwal et al., 2015; Sharma et al., 2016). Moreover, volatile oil, gingerol, alkaloids, and diarylheptanoids were isolated from Ganjiang (Ajish et al., 2015; Li et al., 2016a; Shao et al., 2017). Previous studies have shown that the extracts and compounds of ginger have a variety of pharmacological activities, including thermogenesis (Miyamoto et al., 2015), hypoglycemic activity (Samad et al., 2017), antimicrobial (Ali et al., 2018), antiatherosclerosis (Wang S. et al., 2018), anti-inflammatory (Ho and Chang, 2018), analgesic (Hitomi et al., 2017), anticancer (Kapoor et al., 2016), antioxidant, antiaging functions (Han H. S. et al., 2018; Lee et al., 2019), the effect on the cardiovascular system and energy metabolism, sedative effect, and antiemetic activity (Vishwakarma et al., 2002; Yu et al., 2012; Wen et al., 2019). Correspondingly, Ganjiang is used clinically to treat patients with dizziness, vomiting caused by cold or during pregnancy cough, asthma, etc. (Wang W. X., 2016).

The hot property of Ganjiang can be partially explained by its thermogenic function. For example, Jiang Gui Fang (JG), composed of dried ginger and other TCMs, can increase core temperature, elevate the expressions of UCP1 and PGC- $1 \alpha$ in 
BAT, and enhance the expressions of the lipogenic factor PPAR- $\gamma$ and lipolytic protein hormone-sensitive triglyceride lipase (HSL) in white adipose tissue (WAT). In vitro, silent mating type information regulation 2 homolog 1 (SIRT1) was also enhanced by JG. Taken together, these results indicated that JG can activate BAT and induce browning of WAT via the PPAR $\gamma /$ SIRT1-PGC1 $\alpha$ pathway to increase thermogenesis ( $\mathrm{Zu}$ et al., 2019). In addition, 6-capsaicin, the main active ingredient of Ganjiang, can promote the browning of adipocyte, which is proven by the increase of some brown/beige fat-specific genes such as PGC- $1 \alpha$, Cidea, PR domain containing 16 (Prdm16), gene encoding $\mathrm{Cbp} / \mathrm{p} 300$-interacting transactivator 1 (Cited1), SIRT1, gene encoding transmembrane protein 26 (Tmem26), and Ucp1, as well as the increase of protein including UCP1, PGC- $1 \alpha$, and PRDM16. Some mitochondrial biogenesis markers such as SIRT1 and $p$-AMPK/AMPK are also increased by 6-capsaicin, which illustrates that 6 -capsaicin improves mitochondrial respiration and energy metabolism (Wang et al., 2019).

\section{Rougui}

Rougui is the dried stem bark of Cinnamomum cassia (L.) J.Presl (Lauraceae) and can be used both as a condiment and as a drug. In America, it is used as a food supplement (Wang et al., 2013), while in Asia, it is often used as herbal medicine. In TCM, it is regarded as a Chinese medicine with strong hot property, and it was first recorded in Shennong Bencao Jing and listed as the top grade TCMs (Mamindla et al., 2017; Zhang et al., 2019).

According to the theory of TCMs, Rougui has the functions of warming Yang, dispersing cold to relieve pain, warming channels to promote blood circulation. To treat kidney Yang deficiency, Rougui is combined with other TCMs to enhance kidney Yang. Recorded in Jingyue Quanshu, Yougui Pill, a prescription containing Rougui and other TCMs such as Danggui (the dried root of Angelica sinensis (Oliv.) Diels) and Fuzi, can be used to treat patients with chilly limbs, cold pain in waist and knee, frequent urination, impotence, premature ejaculation, etc. For spleen and kidney Yang deficiency, Guifu Lizhong Wan (recorded in Sanyin Fang) consisting of Rougui, Fuzi, Ginseng (the dried root and rhizome of Panax ginseng C.A.Mey.), Baizhu (the dried rhizome of Atractylodes macrocephala Koidz.), etc. is used to treat symptoms such as cold limbs, decreased appetite, tiredness, and loose stools. For the symptoms of palpitations, shortness of breath, and chest tightness caused by heart Yang deficiency, Rougui is generally used in combination with TCMs such as Ginseng (the dried root and rhizome of Panax ginseng C.A.Mey.) and Huangqi (the dried root of Astragalus mongholicus Bunge) for warming Yang and supplementing qi. Rougui-containing prescriptions also play an important role in the treatment of cold-induced pain and blood stasis syndrome. For example, Guifu Pills consisting of Rougui, Ganjiang (the dried Zingiber officinale Roscoe), and so on have significant effects on treating chest pain and heartache caused by cold invasion, which was recorded in Shoushi Baoyuan. In addition, in Beiji Qianjin Yaofang, Duhuo Jisheng Decoction consisting of Rougui, Danggui (the dried root of Angelica sinensis (Oliv.) Diels), Sangjisheng (the dried twig of Taxillus chinensis (DC.) Danser), etc. has been shown to combat cold and dampness, so it is a commonly considered prescription for the treatment of rheumatism. For women with lumps in the abdomen due to qi stagnation and blood stasis, Peng E Zhu Pill in Jiyin Gangmu, mainly composed of Rougui, Ezhu (the dried rhizome of Curcuma phaeocaulis Valeton), and Taoren (the dried mature seed of Prunus persica (L.) Batsch), is a suitable choice because it can warm channels to promote blood circulation, thereby eliminating lumps.

Extensive research has been carried out on the phytochemical constituents of Rougui. More than 160 components have been isolated and identified from Rougui (Zhang et al., 2019). The chemical components in Rougui are divided into volatile and nonvolatile components, of which the volatile component (volatile oil) is the main active ingredient. Cinnamaldehyde, the main component of volatile oil of Rougui, is regarded as the quality marker of Rougui in Chinese pharmacopoeia (Zhang et al., 2019). In addition, Rougui contains some types of nonvolatile compounds such as polysaccharides (Liu X. et al., 2018), polyphenols (Anderson et al., 2004), and flavonoids (Pardede et al., 2017), and other ingredients such as coumarin (Kim, 2017). The wide range of pharmacological activities of Rougui and its components have been reported, including thermogenesis (Tamura et al., 2012), antioxidant, antiinflammatory, antibacterial, antidiabetic, antiobesity (Song et al., 2017; Lv et al., 2017; Zhu et al., 2017; Wang Y. et al., 2018), antishock (Kwon et al., 2015), antithrombotic (Kontogiorgis et al., 2015), antigastric ulcer effect (Jung et al., 2011), and cardiovascular protection (Gao et al., 2015). Therefore, Rougui is mainly used in the treatment of cardiovascular diseases (Hwa et al., 2012), gastrointestinal diseases (Sebaia et al., 2019), diabetes (Shinjyo et al., 2020), kidney diseases (Zhao et al., 2013b), rheumatic diseases (Chen et al., 2019), gynecological diseases (Sun et al., 2016), etc.

Rougui, as a TCM with hot property, has an important thermogenic effect on human body, and the mechanisms are related to the thermogenic genes in adipose tissue. Kwan et al. showed that Rougui significantly enhanced the expression of UCP1 in 3T3-L1 adipocytes and subcutaneous adipocytes, as well as the expression of other brown adipocyte marker genes such as cell death-inducing DFFA-like effector A (Cidea), $\operatorname{Prdm} 16, \operatorname{PPAR} \gamma, \operatorname{PPAR} \gamma$ coactivator-1 $(P g c)$, and the fatty acid oxidation marker gene carnitine palmitoyltransferase 1 (Cpt1). Rougui also significantly increased the mitochondrial protein biogenesis. Thus, it can induce the browning of subcutaneous adipocyte browning to promote thermogenesis (Shen et al., 2010; Kwan et al., 2017). Further research found that cinnamaldehyde (CA), the main active component of Rougui, activates thermogenesis through PKA/p38 MAPK signaling in subcutaneous adipocyte of mice (Jiang et al., 2017). Specifically, CA can increase the expression of heat production markers such as fibroblast growth factor 21 (Fgf21) and Ucp1, as well as activate PKA and subsequent phosphorylation of p38 MAPK, PKA-dependent phosphorylation of HSL, and lipid droplet-associated protein perilipin 1 (PLIN1). Furthermore, the PKA inhibitor H-89 can significantly block the phosphorylation of PKA substrate, p38 MAPK, HSL, and PLIN1. Correspondingly, the induction of Fgf21 and Ucp1 by CA was significantly inhibited by H-89 (Jiang et al., 2017). 


\section{Important Cold Traditional Chinese Medicines for Hot Syndrome \\ Huanglian}

Huanglian, dried rhizome of medicinal plants from the family Ranunculaceae such as Coptis chinensis Franch., Coptis deltoidea C.Y.Cheng and P.K.Hsiao, and Coptis teeta Wall., has been used in TCM for more than 2000 years (Yi et al., 2013; Qi et al., 2018). According to TCM theory, Huanglian is cold in property and bitter in taste and can enter the meridian of heart, stomach, large intestine, and liver and has the function of clearing away heat and dispelling dampness and detoxifying. It is often used in the treatment of damp-heat syndromes such as diarrhea and vomiting caused by gastrointestinal damp-heat, hot syndromes such as heart and stomach heat, and heat-toxicity syndromes such as carbuncle (Wang et al., 2014).

Specifically, for the hot syndrome of heart that manifested as fever, irritability, even delirium, Huanglian is often combined with the TCMs with the function of clearing heart fire or clearing heat and removing toxicity, such as Huanglian Jiedu Decoction in Waitai Miyao. In the treatment of dysphoria and palpitation caused by hyperactivity of heart fire, Huanglian E-Jiao Decoction in Shanghan Lun, mainly consisting of Huanglian, Ejiao (Colla Corii Asini), and Huangqin (the dried root of Scutellaria baicalensis Georgi), is often preferred. When heart fire is so intense that it causes hematemesis and bleeding, Huanglian is generally used in combination with the TCMs for cooling blood and arresting bleeding, such as Xiexin Decoction in Jingui Yaolüe. In addition, Huanglian also has a strong effect on clearing stomach fire. For example, Qingwei San, a prescription mainly composed of Huanglian, Shigao $\left(\mathrm{CaSO}_{4} \bullet 2 \mathrm{H}_{2} \mathrm{O}\right)$, and other TCMs for clearing stomach heat, has been applied to treat the hot syndromes resulting in stomach fire, such as toothache, redness and swelling of gums, and bleeding of teeth. Of note is that, for the wasting syndrome (Xiao Ke Zheng) caused by stomach heat, including polydipsia, polyuria, polyphagia, emaciation, and fatigue, the powerful effect of Huanglian for clearing stomach fire has attracted much attention. Huanglian was viewed as a curative TCM for patients with wasting syndrome, which was recorded as early as the Wei and Jin Dynasties (1,500 years ago, 220-589 AD). In the subsequent dynasties, many records about Huanglian for the treatment of wasting syndrome were kept in the series of herbal classics. The Хinxiu Bencao in the Tang Dynasty (618-907 AD) pointed out that the Huanglian growing in western China is beneficial to the treatment of wasting syndrome. According to the analysis of Taiping Shenghui Fang (960-1279 A.D.), Huanglian is found to be one of the ten most commonly used TCMs in the treatment of wasting syndrome. Besides, in the Puji Fang completed in the Ming Dynasty around $1406 \mathrm{AD}, 13$ of the 64 formulas for treating wasting syndrome involved Huanglian. The Bencao Gangmu that was published in the same dynasty recorded the treatment of excessive urination, thirst, and emaciation with Huanglian. Therefore, the widespread use of Huanglian for the treatment of wasting syndrome has formed the foundation for its antidiabetic effect. In addition, because of the efficacy of Huanglian in removing damp-heat, clearing heat, and removing toxicity, it is often used to treat dysentery, sores, carbuncles, and furuncles, such as Gegen Huangqin Huanglian Decoction in Shanghan Lun and Huanglian Jiuku Decoction in Waike Zhengzong.

In 1862, berberine (BBR) was first reported as a chemical component isolated from Huanglian (Perrins, 1862). So far, more than 100 chemical components have been isolated and identified from it. Among them, alkaloids, including berberine, palmatine, coptisine, epiberberine, and magnoflorine, are the main active components. In addition to alkaloids, Huanglian also contains chemical components such as organic acids, coumarin, phenylpropanoids, and saccharides (Meng et al., 2018). The wide range of pharmacological effects of the extracts or compounds of Huanglian includes antipyretic, antibacterial, antiviral, antidiabetic, anticancer, anti-inflammatory, and cardiovascular protective effects (Liu et al., 2011; Tan et al., 2016; Hung et al., 2018; Ishikawa et al., 2018; Li et al., 2018; Liang et al., 2019; Kwon and Chan, 2020; Qin et al., 2020). In particular, the pharmacological effects of BBR have been extensively studied. Due to its outstanding antibacterial activity, BBR has been used as an over-the-counter (OTC) drug in the treatment of bacterial diarrhea in China for decades (Kong et al., 2004; Kumar et al., 2015; Jin et al., 2020). Since the early 2000s, BBR has been progressively seen as a potential medicine for hyperlipidemia and diabetes (Dong et al., 2012; Lan et al., 2015; Yao et al., 2015; Cicero and Baggioni, 2016). In addition to metabolic disorders, BBR has constructive effects against cardiovascular diseases, such as heart failure, arrhythmia, thrombosis, hypertension, atherosclerosis, and acute coronary syndrome (Lan et al., 2015; Yao et al., 2015; Li et al., 2016b; Wang K. et al., 2017).

Evidence for the antipyretic effect of Huanglian can partially account for this cold property of Huanglian. As a representative of heat-clearing drug, Huanglian appears in many classic prescriptions for heat-clearing. BBR, the main biologically active component of Huanglian (Zou et al., 2017), could inhibit the formation of arterial plaque and reduce the inflammatory response of aortic tissue in the atherosclerosis rats with damp-heat syndrome (Ke et al., 2020), which may be related to its antipyretic activity. As early as in 1971, Sabir et al. have studied the antipyretic effect of BBR by using a model of experimentally induced fever in rats (Sabir and Bhide, 1971). The antipyretic activity of BBR was also shown by another famous fever model, Brewer's yeast-induced pyrexia mice, and the result was in line with previously reported by Sabir et al. (Küpeli et al., 2002). In order to further understand the potential mechanism of BBR in regulating body temperature, a recent study comprehensively investigated the effect of BBR on environmentally dependent thermogenesis in mice (Jiang et al., 2013). These results showed that BBR can antagonize increasing core body temperatures in hot environments, and further research showed that it limited the expression of HSP70 (heat shock protein 70) and TNF $\alpha$ (tumor necrosis factor, a proinflammatory cytokine) and in mice, suggesting that the antipyretic mechanism of BBR is related to heat shock protein and inflammatory cytokines (Jiang et al., 2013). In addition, Kong et al. demonstrated that Huanglian exerted antipyretic effect on yeast-induced pyrexia rats by regulating the expression of 
transient receptor potential vanilloid 1 (TRPV1, the activation of this protein will cause a burning sensation) and transient receptor potential melastatin 8 (TRPM8, a protein with the function of sensing cold). Specifically, Huanglian can inhibit febrile mediators such as prostaglandin E2 (PGE2) and cyclic adenosine $3^{\prime}, 5^{\prime}$-monophosphate (cAMP), increase antipyretics such as arginine vasopressin (AVP) to activate TRPM8, and inhibit TRPV1 in hypothalamic paraventricular nucleus and supraoptic nucleus, thus exerting antipyretic effect (Kong et al., 2014; Wan et al., 2014).

\section{Dahuang}

Rheum palmatum L., Rheum tanguticum (Maxim. ex Regel) Balf., and Rheum officinale Baill. (Polygonaceae) are endemic species to China (Zhou et al., 2014). Dahuang is their dried root and rhizome and is first recorded in Shennong Bencao Jing. As a representative TCM with cold property, Dahuang has obvious functions of relaxing bowels, clearing heat, detoxification, and cooling blood for hemostasis (Zhang R. Z. et al., 2015), and it has been widely used for cathartic, febrifugal, antidotal, and hemostatic purposes (Lee et al., 2003).

On the one hand, Dahuang can defecate stools and remove food that has accumulated in the gastrointestinal tract; on the other hand, it can clear away heat. Therefore, it is the representative TCM of Hanxia Fa (cold purgative method) (Xie, 2017). In Shanghan Lun, 113 of the 397 formulas mentioned the purgative method and 15 of the 113 formulas used Dahuang. And 20 prescriptions in Jingui Yaolüe selected Dahuang. In addition, more than 30 syndromes registered in Wenyi Lun can be treated by the purgative method, and Dahuang is used as the king TCM in these treatment programs. For instance, Dachengqi Decoction in Shanghan Lun selects Dahuang to treat constipation caused by heat accumulation. Dahuang in Yinchenhao Decoction helps to clear heat and treat jaundice induced by damp-heat, which is recorded in Shanghan Lun. In Jingui Yaolüe, the Xiexin Decoction consisting of Dahuang, Huanglian (the dried rhizome of Coptis chinensis Franch.), and Huangqin (the dried root of Scutellaria baicalensis Georgi) has an important effect on clearing away heat and cooling blood for stopping bleeding. Thereby, it has a good therapeutic effect on patients with hematemesis, bleeding, and hemoptysis. The Dahuang Mudan Decoction composed of Dahuang, Baishao (the dried root cortex of Paeonia lactiflora Pall.), etc. has the outstanding effect of clearing heat and detoxification, so it can treat abscess due to heat exuberance, which has been recorded in Jingui Yaolüe.

The main effective components of Dahuang are anthraquinones such as emodin, emodin methyl ether, aloe emodin, rhein, chrysophanol, etc. In addition, phenolic compounds were identified including catechins, glucose gallates, naphthalenes, sennosides, and stilbenes (Zargar et al., 2011; Pandith et al., 2014; Pandith et al., 2018). According to reports, the crude extracts and isolated compounds of Dahuang have a wide spectrum of pharmacological activities including antipyretic, antibacterial, antifungal, antiviral, anti-inflammatory, antioxidant, anticancer, hypoglycemic, immune-enhancing, liver-protective, kidney-protective, and central neuroprotective effect (Zargar et al., 2011; Kong et al., 2014; Esposito et al., 2016; Li X. et al., 2019). Dahuang's medicinal history can be traced back to a long time ago, and it is known to be effective in treating nearly 57 different diseases (Rokaya et al., 2012). The extracts from Dahuang roots, skins, and leaves have been used as laxatives to help patients ameliorate constipation since ancient times (Wang et al., 2007). In addition, it can also be used in the prevention and treatment of inflammation, tinnitus, bleeding, pain, dysmenorrhea, tumors, diabetes, gastric ulcers, Parkinson's disease, Alzheimer's disease, depression, and severe acute respiratory syndrome (SARS) (Lakshmi, 2016; Li X. et al., 2019).

Notably, previous studies reported that emodin, a principle compound from Dahuang, could regulate cold- and heatsensitive transient receptor potential (TRP) channels. In particular, emodin could significantly increase the mRNA expression of TRPM8 and downregulate TRPV1 mRNA under hot conditions with $39^{\circ} \mathrm{C}$ in cultured dorsal root ganglion (DRG) neurons (Sui et al., 2010a; Sui et al., 2010b). Moreover, a study to explore the possible mechanism of Dahuang's antipyretic effect on yeast-induced pyrexia rats found that Dahuang reduced rectal temperature; importantly, it inhibited the fever-induced expression of TRPV1 and enhanced TRPM8 in hypothalamus and DRG. In addition, endogenous pyrogen (PGE2 and CAMP) and antipyretics (AVP) were restored to a balanced state via the treatment of Dahuang (Kong et al., 2014). These findings provide a new perspective for the role of Dahuang with cold property in pyrexia-related disease.

\section{Huangqin}

Huangqin (the dried root of Scutellaria baicalensis Georgi, Lamiaceae), one of the most widely used TCMs, has been prescribed to treat a variety of diseases in many oriental countries, including North Korea, Mongolia, Japan, and Russia (Zhao et al., 2019). One of the characteristics of Huanqin is that it is cold in property according to the theory of TCMs and has the functions of removing the damp-heat, clearing heat, and removing toxicity, stopping bleeding, and tranquilizing fetus (Committee of National Pharmacopoeia, 2015).

According to the cold property and functions of Huangqin, it is a very appropriate choice therapy for patients with hot syndromes such as damp-heat syndrome, heat-toxicity syndrome, and bleeding syndrome. These hot syndromes specifically include symptoms such as gonorrhea, diarrhea, jaundice, cough caused by lung heat, sore throat, hematemesis, hematochezia, and hematuria (Committee of National Pharmacopoeia, 2015). For the treatment of these hot syndromes, Huangqin plays an indispensable role in a number of formulations, which is recorded in many world-renowned monographs of TCM. Huangqin, for instance, exists potent effects on body heat, diarrhea, and dry mouth caused by damp-heat, which comes from Gegen Qinlian Decoction in Shanghan Lun. Furthermore, Huanglian can also be used to treat a variety of organ dampness-heat syndromes and is especially good at treating cough caused by lung heat. For example, Qingjin Pill in Danxi Xinfa is effective for cough and 
sore throat with lung heat only by using Huangqin. The cold property of Huangqin can help stop bleeding, so it has a prominent effect on bleeding syndrome caused by hot. Huangqin San composed only of Huangqin can achieve the effect of treating hematemesis and bleeding, which is recorded in Taiping Shenghui Fang. Nowadays, Huangqin has been officially registered in Chinese Pharmacopoeia (2015), British Pharmacopoeia (BP 2018), and European Pharmacopoeia (EP 9.0). It is the crucial TCM of numerous renowned traditional Chinese patent medicines and simple preparations, such as Gegen Qinlian Pills (used to treat diarrhea, dysentery, influenza, and fever), Qingqi Huatan Pills (used to treat cough and sore throat caused by lung heat), Yinzhihuang Granules (used to treat jaundice and hepatitis), and Yiqing Capsule (used to treat constipation, pharyngitis, tonsillitis, and gingivitis) (Wang Z. L. et al., 2018).

To date, more than 130 compounds have been isolated from Huangqin, most of which are small molecule compounds and a small number of them are polysaccharides. The small molecules can be divided into four types of structures, namely, free flavonoids, flavonoid glycosides, phenylethanoid glycosides, and other small molecules. Among them, the most representative compounds are flavonoids, including baicalin, baicalein, wogonoside, and wogonin (Wang Z. L. et al., 2018). Moreover, the compounds and extracts isolated from Huangqin possess a wide range of pharmacological activities, including antipyretic, antibacterial, antiviral, antioxidative, anticancer, anticonvulsant, anti-inflammatory, antidiabetic, antidepressant, and hepatoprotective effects (Lin et al., 1980; Konoshima et al., 1992; Park et al., 2007; Wang Z. L. et al., 2018; Kong et al., 2019; Fang et al., 2020; Limanaqi et al., 2020). And it is suitable for the treatment of hepatitis, diarrhea, vomiting, hypertension, hyperglycemia, type 2 diabetes, hyperlipidemia, obesity, and nonalcoholic fatty liver (Committee of National Pharmacopoeia, 2015; Fang et al., 2020).

Targeting the pharmacological effects of Huangqin, of note is that the antipyretic effect of it can partially explain its cold property. Just as early as 1980, Lin et al. investigated the effect of Huangqin on thermoregulation in rats and found that intragastric administration of its extract showed a dosedependent decrease in rectal temperature. And according to the increase in skin temperature, it is estimated that the decrease in body temperature after the application of Huangqin may be caused by skin vasodilation (Lin et al., 1980). Then, Tsai et al. investigated the antipyretic effects of baicalin on rabbits with fever caused by lipopolysaccharide, and the results showed that baicalin may exert its antipyresis by suppressing circulating TNF $a$ accumulation and $N$-methyl-Daspartate (NMDA) receptor-dependent hydroxyl radical pathways in the hypothalamus (Tsai et al., 2006). Furthermore, PGE2 and cAMP, as important neural mediators of fever, also participate in the antipyretic mechanism of baicalin. It was found that baicalin can reverse the change of fever on the contents of PGE2 and cAMP in hypothalamus, and correlation analysis indicated that the contents of PGE2 and cAMP had positive correlations with body temperature in rats (Zhao et al., 2002).

\section{Section Conclusion}

In this section, we introduced the important TCMs with hot property or cold property and their scientific applications. Firstly, we introduced their traditional usage recorded in ancient books and summarized them in Table 1 . These traditional usages of typical hot and cold TCMs show the application principle of the theory of cold and hot properties; that is, the TCMs with hot property can be used to treat patients with cold syndrome and the TCMs with cold property can be used to treat patients with hot syndrome. And we found that enhancing Yang is the common traditional function of hot TCMs, and clearing heat is the common traditional function of cold TCMs. Then, based on modern research, we summarized their phytochemistry, pharmacological activities, and clinical applications (Table 2). We found that although the compounds of each cold TCM are different, all these representative cold TCMs show antipyretic effect. Similarly, although the components of each TCM are different, all these typical hot TCMs have a promoting effect on thermogenesis. In addition, the mechanisms related to the cold and hot properties of these important TCMs were summarized. Specifically, the mechanism of thermogenesis-promoting effect of these hot TCMs mainly included 1) activating of BAT and browning WAT; 2) promoting energy metabolisms. The BAT activation and WAT browning were the common mechanism of thermogenesis-promoting effect of these hot TCMs. As shown in Figure 3, these hot TCMs activated BAT and induced browning of WAT through the PPAR $\gamma$ (SIRT1-PGC1 $\alpha$ ) pathway, thereby promoting thermogenesis. Similarly, the antipyretic mechanism of these cold TCMs mainly included 1) activating TRPM8 and inhibiting TRPV1;2) inhibiting proinflammatory cytokines. The common mechanism of antipyretic of these cold TCMs is associated with activation of TRPM8 and inhibition of TRPV1 (Figure 4).

After summarizing the traditional function and modern biological mechanism of TCMs, we found the following relationships: the traditional function of enhancing Yang of hot TCMs corresponds to thermogenesis-promoting effect. For example, Fuzi (hot in property) can promote thermogenesis by increasing the UCP1 level in BAT, thereby improving hypothermia caused by cold exposure. Similarly, the traditional function of clearing heat of cold TCMs corresponds to antipyretic effect. For example, Dahuang (cold in property) exerts antipyretic effect by activating TRPM8 and inhibiting TRPV1, so as to improve the hot syndrome induced by yeast in rats. These modern biological mechanism studies provide scientific basis for the traditional function of cold and hot TCMs.

\section{PERSPECTIVES}

Even though different TCMs may share the same cold property or hot property, the specific mechanisms behind their cold or hot properties are still different. For example, both Fuzi and Rougui are hot TCMs; however, they cannot be used interchangeably in the clinic in most cases. A previous study based on network pharmacology to explore the mechanism of Fuzi and Rougui in the treatment of cardiocerebral vascular diseases showed that, 
TABLE 1 | The traditional usage of major TCMs with hot or cold property.

\begin{tabular}{|c|c|c|c|}
\hline TCMs name & Property & Traditional usage & References \\
\hline Fuzi & Hot & $\begin{array}{l}\text { Revive Yang for resuscitation, for prostration, cold sweat, and cold limbs, as well as weak and impalpable pulse } \\
\text { Invigorate qi and restore Yang, for energy loss, cold extremities, sweating, plus feeble, and impalpable pulse } \\
\text { Strengthen spleen Yang, for deficient cold in spleen and stomach, cold pain in the abdomen, vomiting, diarrhea, and } \\
\text { cold in limbs } \\
\text { Restore kidney Yang, for dysuria and swelling of the body } \\
\text { Dispel coldness and unlock channels, for wind-cold-dampness arthralgia }\end{array}$ & $\begin{array}{l}\text { Bencao Jingdu } \\
\text { Shanghan Lun } \\
\text { Shengji Zonglu } \\
\text { Taiping Huimin Heji } \\
\text { Jufang }\end{array}$ \\
\hline Ganjiang & Hot & $\begin{array}{l}\text { Restore Yang, for Yang depletion syndrome such as prostration, deadly cold hand and foot, and weak pulse caused } \\
\text { by the Yang deficiency } \\
\text { Invigorate spleen and disperse cold, for deficiency of spleen and stomach, cold pain of epigastric abdomen, loss of } \\
\text { appetite, reduced diet, vomiting, and diarrhea } \\
\text { Warming interior, for dispersing cold and relieve pain } \\
\text { Warm lung and resolve fluid retention, for cough and asthma caused by cold, cold in the back, and phlegm retention }\end{array}$ & $\begin{array}{l}\text { Shanghan Lun } \\
\text { Jingui Yaolüe } \\
\text { Heji Ju Fang }\end{array}$ \\
\hline Rougui & Hot & $\begin{array}{l}\text { Enhance spleen and kidney Yang, for chilly limbs, cold pain in waist and knee, frequent urination, impotence, } \\
\text { premature ejaculation, decreased appetite, tiredness, and loose stools } \\
\text { Strengthen heart Yang, for palpitations, shortness of breath, and chest tightness } \\
\text { Dispel cold and relieve pain, for chest pain, heartache, and rheumatism } \\
\text { Warm channels to promote blood circulation, for abdominal lumps in women }\end{array}$ & $\begin{array}{l}\text { Jingyue Quanshu } \\
\text { Sanyin Fang } \\
\text { Shoushi Baoyuan } \\
\text { Beiji Qianjin Yaofang } \\
\text { Jiyin Gangmu }\end{array}$ \\
\hline Huanglian & Cold & $\begin{array}{l}\text { Clear heart fire, for fever, irritability, delirium, dysphoria, palpitation, hematemesis, and bleeding } \\
\text { Clear stomach fire, for toothache, redness and swelling of gums, bleeding of teeth, polydipsia, polyuria, polyphagia, } \\
\text { emaciation, and fatigue } \\
\text { Remove damp-heat, clear heat, and remove toxicity, for dysentery, sores, carbuncles, and furuncles }\end{array}$ & $\begin{array}{l}\text { Waitai Miyao } \\
\text { Shanghan Lun } \\
\text { Jingui Yaolüe } \\
\text { Taiping Shenghui Fang } \\
\text { Puji Fang } \\
\text { Bencao Gangmu } \\
\text { Waike Zhengzong }\end{array}$ \\
\hline Dahuang & Cold & $\begin{array}{l}\text { Defecate stools and remove food, for constipation because of heat accumulation } \\
\text { Clear heat, for jaundice caused by damp-heat } \\
\text { Cool blood for stopping bleeding, for hematemesis, bleeding, and hemoptysis } \\
\text { Clear heat and remove toxicity, for abscess with a pattern of suppuration due to heat exuberance }\end{array}$ & $\begin{array}{l}\text { Shanghan Lun } \\
\text { Jingui Yaolüe } \\
\text { Wenyi Lun }\end{array}$ \\
\hline Huangqin & Cold & $\begin{array}{l}\text { Remove damp-heat, for body heat, diarrhea, dry mouth, dysentery, influenza, jaundice, and hepatitis } \\
\text { Clear lung heat, for cough and sore throat caused by lung heat } \\
\text { Clear away hot, cool blood, and stop bleeding, for bleeding syndrome caused by hot such as hematemesis and } \\
\text { hemorrhage }\end{array}$ & $\begin{array}{l}\text { Shanghan Lun } \\
\text { Danxi Xinfa } \\
\text { Taiping Shenghui Fang }\end{array}$ \\
\hline
\end{tabular}

TCMs, traditional Chinese medicines.

among the 194 targets of Fuzi and Rougui, 45 targets are shared; that is, three-quarters of the targets are different (Li C. et al., 2020). In addition, from a metabolic perspective, Fuzi can promote energy metabolism by increasing the activity of $\mathrm{Na}^{+}$$\mathrm{K}^{+}$-ATPase and $\mathrm{Ca}^{2+}-\mathrm{Mg}^{2+}$-ATPase enzymes in hepatocytes, which is closely related to thermogenesis (Yu et al., 2012). However, Rougui shows a significant inhibitory effect on $\alpha$-glucosidase, thereby destroying carbohydrate metabolism and ultimately exerting antidiabetic effects (Shihabudeen et al., 2011). Those studies indicated that the specific mechanisms of the hot property of Fuzi and Rougui are different. Even though progress has been made in revealing this difference, more and more studies are still needed to further identify the specific differences in mechanisms.

In recent years, TCM network pharmacology, first proposed and established by Li et al. (2007), has made a great contribution to the study of hot and cold properties of TCMs including the identification of the cold and hot properties of TCMs and the study of cold and hot syndromes or formulas (Li et al., 2007; Li, 2009; Li et al., 2010; Li and Zhang, 2013; Zhang et al., 2013; Li et al., 2014; Zhang Y. Q. et al., 2016; Zheng et al., 2018). As we all know, in network pharmacology research, animal and human experiments are needed to verify the targets and connections. In animal experiments, metabolomics and other omic tools can be used as powerful means to verify the related targets and connections. Omic tools such as metabolomics have become important techniques for studying the responses of complex organisms to drug or other stimuli. The omic tools can provide complementary information for exploring overall biological function by identifying potential targets, which is consistent with the holistic view of TCM (Wang M. et al., 2017). Therefore, the integration of network pharmacology and omic tools such as metabolomics may be a good strategy to study the mechanism of the effects of cold and hot TCMs.

In addition, gut microbiota has emerged as a new Frontier to understand TCMs (Liu J. et al., 2018; Feng et al., 2018; Feng et al., 2019; Feng et al., 2020). Recent studies have shown that cold or hot exposure can result in marked changes in the composition of gut microbiota and a recent paper also hypothesized that the reshaping of the gut microbiota might serve as the main driver for potentiating energy conservation (Chevalier et al., 2015; Worthmann et al., 2017; Zhang et al., 2018; Li B. et al., 2019; Bo et al., 2019). Akkermansia muciniphila is a bacterium that has received a lot of attention because of its health promotion effect. Studies have demonstrated that Akkermansia muciniphila, as an energy sensor, is positively related to the markers of the beginning, like uncoupling protein-1 (UCP-1) (Derrien et al., 2008; Everard et al., 2013; Schneeberger et al., 2015; Liu et al., 
TABLE 2 | The main compounds, pharmacological effects, and clinical applications of major TCMs with hot or cold property.

\begin{tabular}{|c|c|c|c|}
\hline $\begin{array}{l}\text { TCMs } \\
\text { name }\end{array}$ & Property & Major compounds & $\begin{array}{c}\text { Pharmacological } \\
\text { effects }\end{array}$ \\
\hline Fuzi & Hot & $\begin{array}{l}\text { Aconitine, mesaconitine, } \\
\text { hypaconitine, benzoylmesaconine, } \\
\text { benzoylaconitine, } \\
\text { benzoylhypacoitine, flavonoids, } \\
\text { glucide, saponins, glycosides, fatty } \\
\text { acids, ceramides, and uracil }\end{array}$ & $\begin{array}{l}\text { Thermogenesis, the effects on the } \\
\text { cardiovascular system, energy } \\
\text { metabolism, kidney protection, } \\
\text { immune system, anti-inflammation } \\
\text { and analgesic action, and antitumor, } \\
\text { hypoglycemic, and hypotensive } \\
\text { effects }\end{array}$ \\
\hline
\end{tabular}

Ganjiang Hot Volatile oils, anthocyanins, tannins, and spicy compounds, namely gingerol, sesquiterpenes and shogaols, alkaloids, and diarylheptanoids
Rougui Hot

Huanglian Cold

Dahuang Cold

Huangqin Cold
Alkaloids such as berberine, palmatine, coptisine, epiberberine, and magnoflorine, as well as organic acids, coumarin, phenylpropanoids, and saccharides
References polysaccharides, polyphenols, such as coumarin
Thermogenesis, hypoglycemic activity, antiatherosclerosis, antiinflammatory, analgesic, anticancer, antioxidant, and antiaging functions, and the effect on the cardiovascular system and energy metabolism, sedative effect, and antiemetic activity
Dizziness, vomiting caused by cold, vomiting during pregnancy, cough, and asthma
Antipyretic, antibacterial, antiviral, antidiabetic, anticancer, antiinflammatory, and cardiovascular protective effects
Bacterial-caused diarrhea, hyperlipidemia, diabetes, heart failure, arrhythmia, thrombosis hypertension, atherosclerosis, and acute coronary syndrome
Cardiovascular diseases, gastrointestinal diseases, diabetes, kidney diseases, rheumatic diseases, and gynecological diseases
Shock, hypotension, coronary heart disease, chronic heart neuralgia, joint pain, and rheumatoid arthritis antidiabetic, antiobesity, antishock, effect, and cardiovascular protection
Yu et al. (2015), Chen et al. (2008), Tang et al. (2012), Sun et al. (2019), Zhao et al. (2020), Konno et al. (1985), Lyu et al. (2008), Sang et al. (2005), He et al. (2018), Deng et al. (2019), Zhao et al. (2012), Zheng et al. (2014), Tan et al. (2014), Yang et al. (2020), Zhang et al. (2017), Huang et al. (2010), Zhou et al. (2015), Zuo et al. (2015) Chrubasik et al. (2005), Semwal et al. (2015), Sharma et al. (2016), Ajish et al. (2015), Li et al. (2016a), Shao et al. (2017), Miyamoto et al. (2015), Samad et al. (2017), Ali et al., 2018, Wang S. et al. (2018), Ho and Chang (2018), Hitomi et al. (2017), Kapoor et al. (2016), Han H. S. et al. (2018), Lee et al. (2019), Vishwakarma et al. (2002), Yu et al. (2012), Wen et al. (2019), Wang W. X. (2016) Zhang et al. (2019), Liu X. et al. (2018), Anderson et al. (2004), Pardede et al. (2017), Kim (2017), Tamura et al. (2012), Lv et al. (2017), Wang Y. et al. (2018), Zhu et al. (2017), Kwon et al. (2015), Kontogiorgis et al. (2015), Jung et al. (2011), Gao et al. (2015), Hwa et al. (2012), Sebaia et al. (2019), Shinjyo et al. (2020), Zhao et al. (2013b), Chen et al. (2019), Sun et al. (2016)

Perrins (1862), Meng et al. (2018), Tan et al. (2016), Li et al. (2018), Ishikawa et al. (2018), Qin et al. (2020), Kwon and Chan (2020), Hung et al. (2018), Liu et al. (2011), Kumar et al. (2015), Jin et al. (2020), Kong et al. (2004), Dong et al. (2012), Yao et al. (2015), Cicero and Baggioni (2016), Lan et al. (2015), Yao et al. (2015), Li et al. (2016b), Wang K. et al. (2017)

Zargar et al. (2011), Pandith et al. (2014), Pandith et al. (2018),
Antipyretic, antibacterial, antifungal, antiviral, anti-inflammatory, antioxidant, anticancer, hypoglycemic, immune-enhancing, as well as protecting liver, kidney, and central nervous

Antipyretic, antibacterial, antiviral, antioxidant, anticancer, anticonvulsant, anti-inflammatory, antidiabetic, antidepressant, and liver protection
Constipation, inflammation, tinnitus, bleeding, pain, dysmenorrhea, tumors, diabetes, gastric ulcers, Parkinson's disease, Alzheimer's disease, depression, and severe acute respiratory syndrome Hepatitis, diarrhea, vomiting, hypertension, hyperglycemia, type 2 diabetes, hyperlipidemia, obesity, and nonalcoholic fatty liver
Kong et al. (2014), Esposito et al. (2016), Li X. et al. (2019), Rokaya et al. (2012), Wang et al. (2007), Lakshmi (2016)

Wang Z. L. et al. (2018), Lin et al. (1980), Konoshima et al. (1992), Park et al. (2007), Kong et al. (2019), Limanaqi et al. (2020), Fang et al. (2020), Committee of National Pharmacopoeia (2015)

TCMs, traditional Chinese medicines.

Major compounds refer to the compounds that have a relatively large percentage in TCMs and have been extensively studied in pharmacology. 


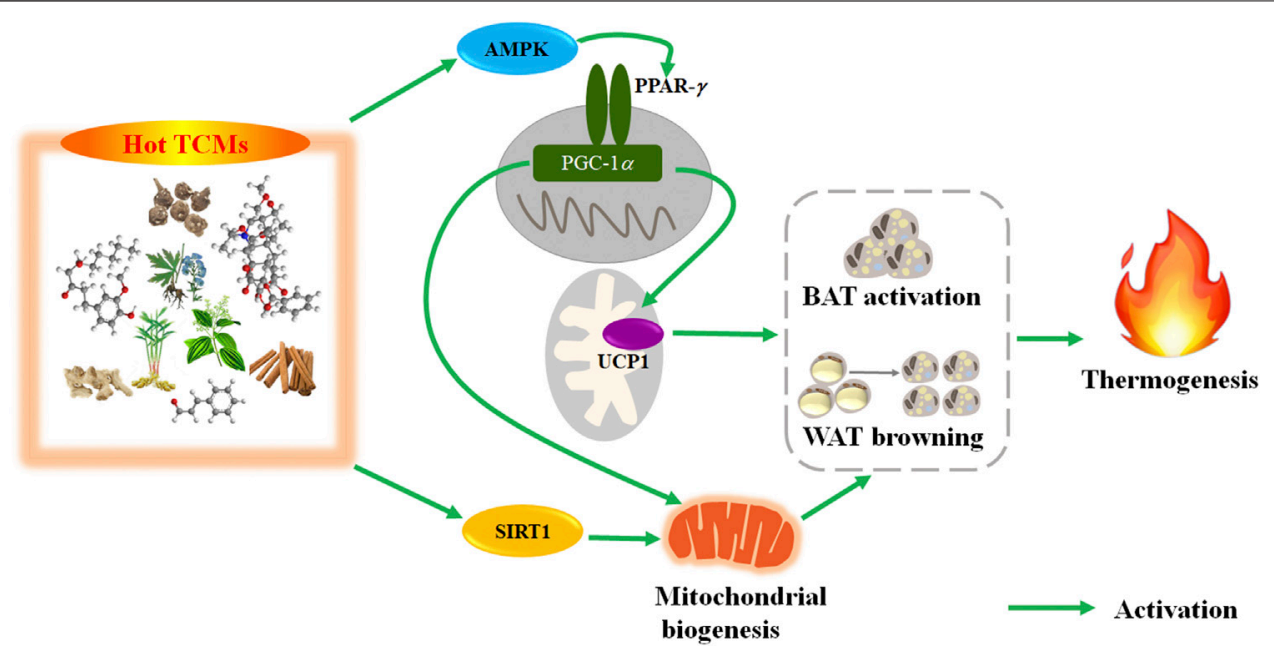

FIGURE 3 The paradigm summarizes the thermogenesis mechanism of hot TCMs via activating BAT and browning WAT. TCMs, traditional Chinese medicines; PPAR- $\gamma$, peroxisome proliferator-activated receptor-gamma; PGC-1 $\alpha$, peroxisome proliferator-activated receptor-gamma coactivator 1-alpha; UCP1, uncoupling protein 1; SIRT1, silent mating type information regulation 2 homolog 1; BAT, brown adipose tissue; WAT, white adipose tissue.

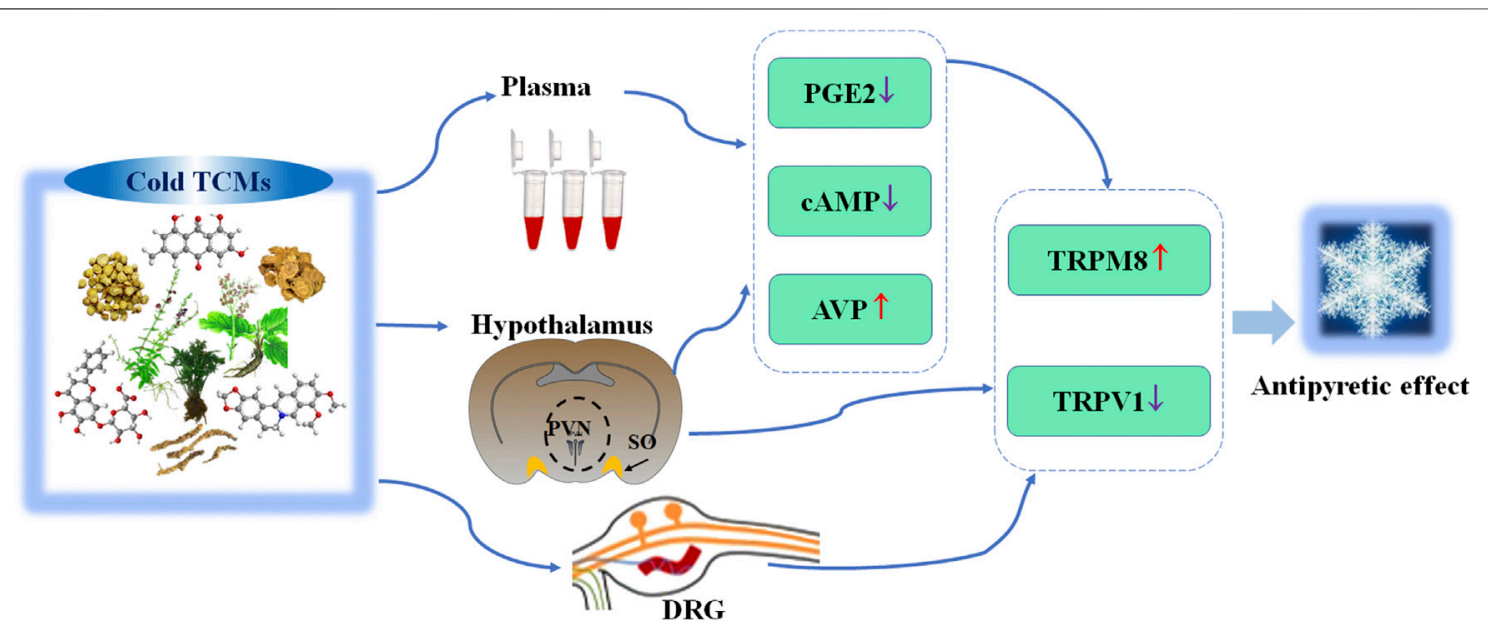

FIGURE 4 | The paradigm summarizes the antipyretic mechanism of cold TCMs via activating TRPV1 and inhibiting TRPM8. TCMs, traditional Chinese medicines; PVN, paraventricular nuclei; SO, supraoptic nucleus; DRG, dorsal root ganglion; PGE2, prostaglandin E2; Camp, cyclic adenosine $3^{\prime}, 5^{\prime}$-monophosphate; AVP, arginine vasopressin; TRPM8, transient receptor potential melastatin 8; TRPV1, transient receptor potential vanilloid 1; $\uparrow$, increase; $\downarrow$, decrease.

2017). Further evidence indicates that cold exposure changes the diversity and composition of the gut microbiota in mice, resulting in alters in the production of microbial metabolites (Ziętak et al., 2016; Worthmann et al., 2017; Li B. et al., 2019; Wang D. et al., 2020). Among these metabolites, the production of short-chain fatty acids (SCFAs) is found to be significantly increased in cold environments. Moreover, isotope tracing experiments have shown that SCFAs are distributed in multiple tissues (including the brain, WAT, and BAT) and metabolized in the tricarboxylate (TCA) cycle (Zhang et al., 2018; Li B. et al., 2019). Notably, a recent study showed that butyrate (SCFA) increases the thermogenesis in BAT and WAT by activating lysine-specific demethylase 1 (LSD1) (Wang D. et al., 2020). In addition, bile acids (BAs), the metabolites of gut microbiota, play an important role in thermogenesis (Teodoro et al., 2014; Zietak and Kozak, 2016). Recently, research showed that bile acid can shape the gut microbiome and promote adaptive thermogenesis in a cold environment (Worthmann et al., 2017). Taken together, those studies have shown that gut microbiota and gut microbiota metabolites play a key role in regulating thermogenesis during cold or hot exposure. Thus, it can be speculated that the cold and hot properties of TCMs might have an important relationship with gut microbiota metabolites. Correspondingly, the integration of $16 \mathrm{~S}$ rRNA gene sequencing and metabolomics, a 
multiomics combination mode, can be considered in the future to study the cold and hot properties of TCMs.

\section{CONCLUSION}

Cold and hot properties of TCMs have played and will still play important roles in guiding TCM practitioners to combating human diseases. From ancient times to the present, people have been exploring the mystery of cold and hot properties of TCMs. In ancient times, the identification of cold and hot properties mainly depends on the healthy human's direct feeling on a given TCM or the effects of TCMs on patients with cold or hot syndrome. Nowadays, some animal models (such as collagen-induced arthritis rats) and advanced technologies (such as network pharmacology) have been used to identify the cold and hot properties of TCMs. In recent years, phytochemical and pharmacologic research of cold and hot TCMs have indicated that although the chemical components are different, the representative cold TCMs all have antipyretic effects, and the typical hot TCMs all can promote thermogenesis. In addition, the studies on the mechanism related to the cold and hot properties have demonstrated that activating TRPM8 and

\section{REFERENCES}

Ajish, K. R., Antu, K. A., Riya, M. P., Preetharani, M. R., Raghu, K. G., Dhanya, B. P., et al. (2015). Studies on $\alpha$-glucosidase, aldose reductase and glycation inhibitory properties of sesquiterpenes and flavonoids of Zingiber zerumbet Smith. Nat. Prod. Res. 29, 947-952. doi:10.1080/ 14786419.2014.956741

Ali, G., Hawa, J., Ali, B., and Amin, T. M. (2018). Formation of 6-, 8- and 10shogaol in ginger through application of different drying methods: altered antioxidant and antimicrobial activity. Molecules 23, 1646. doi:10.3390/ molecules23071646

Anderson, R. A., Broadhurst, C. L., Polansky, M. M., Schmidt, W. F., Khan, A., Flanagan, V. P., et al. (2004). Isolation and characterization of polyphenol typea polymers from cinnamon with insulin-like biological activity. J. Agric. Food Chem. 52, 65-70. doi:10.1021/jf034916b

Bo, T. B., Zhang, X. Y., Wen, J., Deng, K., Qin, X. W., and Wang, D. H. (2019). The microbiota-gut-brain interaction in regulating host metabolic adaptation to cold in male Brandt's voles (Lasiopodomys brandtii). ISME J. 13, 3037-3053. doi:10.1038/s41396-019-0492-y

Chang, C. P., Chang, J. Y., Wang, F. Y., and Chang, J. G. (1995). The effect of Chinese medicinal herb Zingiberis Rhizoma extract on cytokine secretion by human peripheral blood mononuclear cells. J. Ethnopharmacol. 48, 13-19. doi:10.1016/0378-8741(95)01275-i

Chao, Y. Y., You, E., Chang, Y. P., and Dong, X. (2020). Anxiety symptoms, depressive symptoms, and traditional Chinese medicine use in U.S. Chinese older adults. J. Immigr. Minority Health 22. 746-753. doi:10.1007/s10903-01900935-0

Chen, H., Guo, J., Pang, B., Zhao, L., and Tong, X. (2015). Application of herbal medicines with bitter flavor and cold property on treating diabetes mellitus. Evid. Based. Complement. Alternat. Med. 2015, 529491. doi:10.1155/2015/ 529491

Chen, Z., Zhao, Y., Liu, S., Zheng, Q., Liu, T., Ma, X., et al. (2015). Study on hot property differences of Aconiti Lateralis Radix Praeparata and its compatibility with different ginger processed products based on biothermodynamics. J. Therm. Anal. Calorim. 120, 1043-1051. doi:10.1007/ s10973-014-4032-8 inhibiting TRPV1 are the common mechanisms of the antipyretic effect of cold TCMs, and BAT activation and WAT browning are the common mechanisms of hot TCMs to promote thermogenesis. Although some progress has been made in understanding the cold and hot properties of TCMs, more and more studies are still needed to further explore the mechanism differences of TCMs with the same property.

\section{AUTHOR CONTRIBUTIONS}

WF and CP conceived of and proposed the idea. JL, WF, and CP reviewed literature. JL, WF, and $\mathrm{CP}$ contributed to writing, revising, and proofreading the review. All authors read and approved the final manuscript.

\section{FUNDING}

This work was supported by the National Natural Science Foundation of China (nos. 81891010, 81891012, 81630101, and U19A2010) and Science and Technology Ministry of China (2108ZX09721001-008).

Chen, H., Pan, T., Liu, P., Wang, P., and Xu, S. (2019). Baihu Jia Guizhi Decoction improves rheumatoid arthritis inflammation by regulating succinate/SUCNR1 metabolic signaling pathway. Evid. Based. Complement. Alternat. Med. 2019, 3258572. doi:10.1155/2019/3258572

Chen, J. H., Lee, C. Y., Liau, B. C., Lee, M. R., Jong, T. T., and Chiang, S. T. (2008). Determination of aconitine-type alkaloids as markers in Fuzi (Aconitum carmichaeli) by LC/(+) ESI/MS (3). J. Pharmaceut. Biomed. Anal. 48, 1105-1011. doi:10.1016/j.jpba.2008.08.022

Chen, L. C., Wang, B. R., Chou, Y. C., and Tien, J. H. (2005). Drug utilization pattern of Chinese herbal medicines in a general hospital in taiwan. Pharmacoepidemiol. Drug Saf. 14, 651-657. doi:10.1002/pds.1087

Chen, S. F., Huang, Y. P., Wan, S. C., Huang, Y. X., Liang, H. Q., and Chen, S. D. (2018). Effect of banxia xiexin decoction on helicobacter pylori-related peptic ulcers and its possible mechanism via the TGF- $\beta /$ Smad signaling pathway. J. Tradit. Chin. Med. 38, 419-426. doi:10.1016/S0254-6272(18) 30633-2

Cheng, H. T., Chen, C. R., Li, C. Y., Huang, C. Y., Shu, W. Y., and Hsu, I. C. (2016). The classification of sini decoction pattern in traditional chinese medicine by gene expression profiling. Evid. Based. Complement. Alternat. Med. 2016, 8239817. doi:10.1155/2016/8239817

Cheung, F. (2011). TCM: made in China. Nature. 480, S82-S83. doi:10.1038/ $480 \mathrm{~S} 82 \mathrm{a}$

Chevalier, C., Stojanovic, O., Colin, D. J., Suarez-Zamorano, N., Tarallo, V., Veyrat-Durebex, C., et al. (2015). Gut microbiota orchestrates energy homeostasis during cold. Cell 163, 1360-1374. doi:10.1016/j.cell.2015. 11.004

Chi, X. S., Wang, S. J., Baloch, Z., Zhang, H. X., Li, X. Y., Zhang, Z., et al. (2019). Research progress on classical traditional Chinese medicine formula Lily Bulb and Rehmannia Decoction in the treatment of depression. Biomed. Pharmacother. 112, 108616. doi:10.1016/j.biopha.2019

Chrubasik, S., Pittler, M. H., and Roufogalis, B. D. (2005). Zingiberis Rhizoma: a comprehensive review on the ginger effect and efficacy profiles. Phytomedicine 12, 684-701. doi:10.1016/j.phymed.2004.07.009

Cicero, A. F., and Baggioni, A. (2016). Berberine and its role in chronic disease. Adv. Exp. Med. Biol. 928, 27-45. doi:10.1007/978-3-319-41334-1_2

Committee of National Pharmacopoeia (2015). Pharmacopoeia of people s Republic of China (I) Beijing: Chemical Industry Press, 301. 
Cui, J. X., Cui, H. F., Yang, M. R., Du, S. Y., Li, J. F., Li, Y. X., et al. (2019). Tongue coating microbiome as a potential biomarker for gastritis including precancerous cascade. Protein. Cell. 10, 496-509. doi:10.1007/s13238-0180596-6

Deng, X. H., Liu, J. J., Sun, X. J., Dong, J. C., and Huang, J. H. (2019). Benzoylaconine induces mitochondrial biogenesis in mice via activating AMPK signaling cascade. Acta Pharmacol. Sin. 40, 658-665. doi:10.1038/ s41401-018-0174-8

Derrien, M., Collado, M. C., Ben-Amor, K., Salminen, S., and de Vos, W. M. (2008). The mucin degrader Akkermansia muciniphila is an abundant resident of the human intestinal tract. Appl. Environ. Microbiol. 74, 1646-1648. doi:10.1128/ AEM.01226-07

Dong, H., Wang, N., Zhao, L., and Lu, F. (2012). Berberine in the treatment of type 2 diabetes mellitus: a systemic review and meta-analysis. Evid. Based. Complement. Alternat. Med. 2012, 591654. doi:10.1155/2012/591654

Esposito, F., Carli, I., Del Vecchio, C., Xu, L., Corona, A., Grandi, N., et al. (2016). Sennoside A, derived from the traditional Chinese medicine plant Rheum L., is a new dual HIV-1 inhibitor effective on HIV-1 replication. Phytomedicine 23, 1383-1391. doi:10.1016/j.phymed.2016.08.001

Everard, A., Belzer, C., Geurts, L., Ouwerkerk, J. P., Druart, C., Bindels, L. B., et al. (2013). Cross-talk between Akkermansia muciniphila and intestinal epithelium controls diet-induced obesity. Proc. Natl. Acad. Sci. U.S.A. 110, 9066-9071. doi:10.1073/pnas.1219451110

Fang, P., Yu, M., Shi, M., Bo, P., Gu, X., and Zhang, Z. (2020). Baicalin and its aglycone: a novel approach for treatment of metabolic disorders. Pharmacol. Rep. 72, 13-23. doi:10.1007/s43440-019-00024-x

Fang, W. T., Zhan, Z. L., Peng, H. S., and Huang, L. Q. (2017). Historical evolution and change of differentiation on dried ginger, fresh ginger and baked ginger. China J. Chin. Mater. Med. 42, 1641-1645. doi:10.19540/j.cnki.cjcmm.2017.0065

Feng, W., Ao, H., and Peng, C. (2018). Gut Microbiota, short-chain fatty acids, and herbal medicines. Front. Pharmacol. 9, 1354. doi:10.3389/fphar.2018.01354

Feng, W., Ao, H., Peng, C., and Yan, D. (2019). Gut microbiota, a new Frontier to understand traditional Chinese medicines. Pharmacol. Res. 142, 176-191. doi:10.1016/j.phrs.2019.02.024

Feng, W., Liu, J., Ao, H., Yue, S. J., and Peng, C. (2020). Targeting gut microbiota for precision medicine: focusing on the efficacy and toxicity of drugs. Theranostics. 10, 11278-11301. doi:10.7150/thno.47289

Fu, X. J., Mervin, L. H., Li, X. B., Yu, H. Y., Li, J. Y., Zobir, S. Z. M., et al. (2017). Toward understanding the cold, hot, and neutral nature of Chinese medicines using in silico mode-of-action analysis. J. Chem. Inf. Model. 57, 468-483. doi:10. 1021/acs.jcim.6b00725

Gao, Y., Hao, J., Zhang, H., Qian, G., Jiang, R., Hu, J., et al. (2015). Protective effect of the combinations of glycyrrhizic, ferulic and cinnamic acid pretreatment on myocardial ischemia-reperfusion injury in rats. Exp. Ther. Med. 9, 435-445. doi:10.3892/etm.2014.2134

Guo, Q., Mao, X., Zhang, Y., Meng, S., Xi, Y., Ding, Y., et al. (2016). Guizhishaoyao-zhimu decoction attenuates rheumatoid arthritis partially by reversing inflammation-immune system imbalance. J. Transl. Med. 14, 165. doi:10.1186/ s12967-016-0921-x

Han, H. S., Kim, K. B., Jung, J. H., An, I. S., Kim, Y. J., and An, S. (2018). Antiapoptotic, antioxidant and anti-aging effects of 6-shogaol on human dermal fibroblasts. Biomed. Dermatol. 2, 27. doi:10.1186/s41702-018-0037-4

Han, X. Y., Wang, Y. N., and Dou, D. Q. (2018). Regulatory effects of Poria on substance and energy metabolism in cold-deficiency syndrome compared with heat-deficiency syndrome in rats. Chin. J. Nat. Med. 16, 936-945. doi:10.1016/ S1875-5364(18)30135-3

Harms, M., and Seale, P. (2013). Brown and beige fat: development, function and therapeutic potential. Nat. Med. 19, 1252-1263. doi:10.1038/nm.3361

He, Y. N., Ou, S. P., Xiong, X., Pan, Y., Pei, J., Xu, R. C., et al. (2018). Stems and leaves of Aconitum carmichaelii Debx. as potential herbal resources for treating rheumatoid arthritis: chemical analysis, toxicity and activity evaluation. Chin. J. Nat. Med. 16, 644-652. doi:10.1016/\$1875-5364(18)30104-3

Hitomi, S., Ono, K., Terawaki, K., Matsumoto, C., Mizuno, K., Yamaguchi, K., et al. (2017). 6-gingerol and 6-shogaol, active ingredients of the traditional japanese medicine hangeshashinto, relief oral ulcerative mucositis-induced pain via action on $\mathrm{Na}^{+}$channels. Pharmacol. Res. 117, 288-302. doi:10.1016/j.phrs. 2016.12.026
Ho, S. C., and Chang, Y. H. (2018). Comparison of inhibitory capacities of 6-, 8and 10-gingerols/shogaols on the canonical NLRP3 inflammasome-mediated IL-1 $\beta$ secretion. Molecules. 23, 466. doi:10.3390/molecules 23020466

Hong, H. Y. (2012). Aconitum carmichaelii debeaux and huoshen Pai. Zhejiang J. Tradit. Chin. Med. 11, 843

Huang, X., Tang, J., Zhou, Q., Lu, H., Wu, Y., and Wu, W. (2010). Polysaccharide from Fuzi (FPS) prevents hypercholesterolemia in rats. Lipids Health Dis. 9, 9. doi:10.1186/1476-511X-9-9

Huang, Y., Yao, P., Leung, K. W., Wang, H. Y., Kong, X. P., Wang, L., et al. (2018). The yin-yang property of chinese medicinal herbs relates to chemical composition but not anti-oxidative activity: an illustration using spleenmeridian herbs. Front. Pharmacol. 9, 1304. doi:10.3389/fphar.2018.01304

Hung, T. C., Jassey, A., Lin, C. J., Liu, C. H., Lin, C. C., Yen, M. H., et al. (2018). Methanolic extract of rhizoma coptidis inhibits the early viral entry steps of hepatitis C Virus infection. Viruses. 10, 669. doi:10.3390/v10120669

Hwa, J. S., Jin, Y. C., Lee, Y. S., Ko, Y. S., Kim, Y. M., Shi, L. Y., et al. (2012). 2methoxycinnamaldehyde from Cinnamomum cassia reduces rat myocardial ischemia and reperfusion injury in vivo due to HO-1 induction. J. Ethnopharmacol. 139, 605-615. doi:10.1016/j.jep.2011.12.001

Ishikawa, K., Takahashi, K., Hosoi, S., Takeda, H., Yoshida, H., Wakana, D., et al. (2018). Antimicrobial agent isolated from Coptidis rhizome extract incubated with Rhodococcus sp. strain BD7100. J. Antibiot. 72, 71-78. doi:10.1038/s41429018-0114-3

Jiang, B., Liang, X. J., Chen, Y., Ma, T., Liu, L. Y., Li, J. F., et al. (2012). Integrating next-generation sequencing and traditional tongue diagnosis to determine tongue coating microbiome. Sci. Rep. 2, 936. doi:10.1038/srep00936

Jiang, J., Emont, M. P., Jun, H., Qiao, X., Liao, J., Kim, D. I., et al. (2017). Cinnamaldehyde induces fat cell-autonomous thermogenesis and metabolic reprogramming. Metabolism 77, 58-64. doi:10.1016/j.metabol.2017.08.006

Jiang, J. F., Wang, Y. G., Hu, J., Lei, F., Kheir, M. M., Wang, X. P., et al. (2013). Novel effect of berberine on thermoregulation in mice model induced by hot and cold environmental stimulation. PloS One 8, e54234. doi:10.1371/journal. pone. 0054234

Jin, J., Xu, M., Liu, Y. X., Ji, Z. X., Dai, K. L., Zhang, L., et al. (2020). Alginate-based composite microspheres coated by berberine simultaneously improve hemostatic and antibacterial efficacy. Colloids Surf. B Biointerfaces 194, 111168. doi:10.1016/j.colsurfb.2020.111168

Jung, J., Lee, J. H., Bae, K. H., and Jeong, C. S. (2011). Anti-gastric actions of eugenol and cinnamic acid isolated from cinnamomi ramulus. Yakugaku Zasshi 131, 1103-1110. doi:10.1248/yakushi.131.1103

Kan, D. F., Ji, X. M., Chen, Q., Yu, W. C., and Wu, J. B. (2018). Effect of serum containing Aconite on energy metabolism of rat primary hepatocytes. Liaoning J. Tradit. Chin. Med. 45, 645-647

Kanawong, R., Obafemi-Ajayi, T., Ma, T., Xu, D., Li, S., and Duan, Y. (2012). Automated tongue feature extraction for ZHENG classification in traditional chinese medicine. Evid. Based. Complement. Alternat. Med. 2012, 912852. doi:10.1155/2012/912852

Kapoor, V., Aggarwal, S., and Das, S. N. (2016). 6-Gingerol Mediates its anti-tumor activities in human oral and cervical cancer cell lines through apoptosis and cell cycle arrest. Phytother Res. 30, 588-595. doi:10.1002/ptr.5561

Ke, X., Huang, Y., Li, L., Xin, F., Xu, L., Zhang, Y., et al. (2020). Berberine attenuates arterial plaque formation in atherosclerotic rats with damp-heat syndrome via regulating autophagy. Drug Des. Dev. Ther. 14, 2449-2460. doi:10.2147/DDDT. S250524

Kemler, M. A., Reulen, J. P., Barendse, G. A., van Kleef, M., de Vet, H. C., and van den Wildenberg, F. A. (2001). Impact of spinal cord stimulation on sensory characteristics in complex regional pain syndrome type I: a randomized trial. Anesthesiology 95, 72-80. doi:10.1097/00000542-200107000-00016

Kim, J. H. (2017). Extraction time and temperature affect the extraction efficiencies of coumarin and phenylpropanoids from Cinnamomum cassia bark using a microwave-assisted extraction method. J. Chromatogr. B. Analyt. Technol. Biomed. Life. Sci. 1063, 196-203. doi:10.1016/j.jchromb.2017.08.008

Kong, W., Wei, J., Abidi, P., Lin, M., Inaba, S., Li, C., et al. (2004). Berberine is a novel cholesterol-lowering drug working through a unique mechanism distinct from statins. Nat. Med. 10, 1344-1351. doi:10.1038/nm1135

Kong, X., Wan, H., Su, X., Zhang, C., Yang, Y., Li, X., et al. (2014). Rheum palmatum L. and Coptis chinensis Franch., exert antipyretic effect on yeast- 
induced pyrexia rats involving regulation of TRPV1 and TRPM8 expression. J. Ethnopharmacol. 153, 160-168. doi:10.1016/j.jep.2014.02.007

Kong, Z., Liang, N., Yang, G. L., Zhang, Z., Liu, Y., Li, J., et al. (2019). Xiao Chai Hu Tang, a herbal medicine, for chronic hepatitis B. cochrane. Database. Syst. Rev. 2019, CD013090. doi:10.1002/14651858

Konno, C., Murayama, M., Sugiyama, K., Arai, M., Murakami, M., Takahashi, M., et al. (1985). Isolation and hypoglycemic activity of aconitans A, B, C, and D, glycans of aconitum carmichaeli roots. Planta Med. 51, 160-161. doi:10.1055/s2007-969436

Konoshima, T., Kokumai, M., Kozuka, M., Iinuma, M., Mizuno, M., Tanaka, T., et al. (1992). Studies on inhibitors of skin tumor promotion. XI. inhibitory effects of flavonoides from Scutellaria baicalensis on epstein-barr virus activation and their anti-tumor-promoting activities. Chem. Pharm. Bull. 40, 531-533. doi:10.1248/cpb.40.531

Kontogiorgis, C., Nicolotti, O., Mangiatordi, G. F., Tognolini, M., Karalaki, F., Giorgio, C., et al. (2015). Studies on the antiplatelet and antithrombotic profile of anti-inflammatory coumarin derivatives. J. Enzym. Inhib. Med. Chem. 30, 925-933. doi:10.3109/14756366.2014.995180

Kumar, A., and Ekavali, Chopra, K., Mukherjee, M., Pottabathini, R., and Dhull, D. K. (2015). Current knowledge and pharmacological profile of berberine: an update. Eur. J. Pharmacol. 761, 288-297. doi:10.1016/j. ejphar.2015.05.068

Küpeli, E., Koşar, M., Yeşilada, E., Hüsnü, K., and Başer, C. (2002). A comparative study on the anti-inflammatory, antinociceptive and antipyretic effects of isoquinoline alkaloids from the roots of turkish berberis species. Life Sci. 72, 645-657. doi:10.1016/s0024-3205(02)02200-2

Kwan, H. Y., Wu, J., Su, T., Chao, X. J., Liu, B., Fu, X., et al. (2017). Cinnamon induces browning in subcutaneous adipocytes. Sci. Rep. 7, 2447. doi:10.1038/ s41598-017-02263-5

Kwon, H., Lee, J. J., Lee, J. H., Cho, W. K., and Jin, Y. M. (2015). Cinnamon and its components suppress vascular smooth muscle cell proliferation by upregulating cyclin-dependent kinase inhibitors. Am. J. Chin. Med. 43, 621-636. doi:10.1142/S0192415X1550038X

Kwon, S., and Chan, A. T. (2020). Extracting the benefits of berberine for colorectal cancer. Lancet. Gastroenterol. Hepatol. 5, 231-233. doi:10.1016/S2468-1253(19) 30430-3

Lai, L., Flower, A., Prescott, P., Wing, T., Moore, M., and Lewith, G. (2017). Standardised versus individualised multiherb chinese herbal medicine for oligomenorrhoea and amenorrhoea in polycystic ovary syndrome: a randomised feasibility and pilot study in the UK. BMJ. Open. 7, e011709. doi:10.1136/bmjopen-2016-011709

Lakshmi, V. (2016). Potent gastroprotective effect chrysophanol and emodin from Rehum Emodi via $\mathrm{H}^{+} \mathrm{K}^{+}$-Atpase inhibition and increasing the Pge2 level in rats. Nat. Prod. 12, 1-11.

Lan, J., Zhao, Y., Dong, F., Yan, Z., Zheng, W., Fan, J., et al. (2015). Meta-analysis of the effect and safety of berberine in the treatment of type 2 diabetes mellitus, hyperlipemia and hypertension. J. Ethnopharmacol. 161, 69-81. doi:10.1016/j. jep.2014.09.049

Lee, E. B., Kim, J. H., Kim, Y. J., Noh, Y. J., Kim, S. J., Hwang, I. H., et al. (2019). Lifespan-extending property of 6-shogaol from zingiber officinale roscoe in caenorhabditis elegans. Arch. Pharm. Res. 42, 370. doi:10.1007/s12272-0181052-0

Lee, J. H., Kim, J. M., and Kim, C. (2003). Pharmacokinetic analysis of rhein in Rheum undulatum L. J. Ethnopharmacol. 84, 5-9. doi:10.1016/s0378-8741(02) 00222-2

Li, A. R., Li, A. Q., Deng, Z. J., Guo, J. W., and Wu, H. K. (2020). Cross-species annotation of expressed genes and detection of different functional gene modules between 10 cold- and 10 hot-propertied Chinese herbal medicines. Front. Genet. 11, 532. doi:10.3389/fgene.2020.00532

Li, C., Du, X., Liu, Y., Liu, Q. Q., Zhi, W. B., Wang, C. L., et al. (2020). A systems pharmacology approach for identifying the multiple mechanisms of action for the rougui-fuzi herb pair in the treatment of cardiocerebral vascular diseases. Evid. Based. Complement. Alternat. Med. 2020, 5196302. doi:10.1155/2020/ 5196302

Li, B., Li, L., Li, M., Lam, S. M., Wang, G., Wu, Y., et al. (2019). Microbiota depletion impairs thermogenesis of brown adipose tissue and browning of white adipose tissue, Cell Rep. 26, 2720-2737. doi:10.1016/j.celrep.2019.02.015
Li, X., Chu, S., Liu, Y., and Chen, N. (2019). Neuroprotective effects of anthraquinones from Rhubarb in central nervous system diseases. Evid. Based. Complement. Alternat. Med. 2019, 3790728. doi:10.1155/2019/3790728

Li, C. L., Tan, L. H., Wang, Y. F., Luo, C. D., Chen, H. B., Lu, Q., et al. (2018). Comparison of anti-inflammatory effects of berberine, and its natural oxidative and reduced derivatives from Rhizoma Coptidis in vitro and in vivo. Phytomedicine. 52, 272-283. doi:10.1016/j.phymed.2018.09.228

Li, H. Y., Zhao, L. H., Zhang, B., Jiang, Y. Y., Wang, X., Guo, Y., et al. (2014). A network pharmacology approach to determine active compounds and action mechanisms of ge-gen-qin-lian decoction for treatment of type 2 diabetes. Evid. Based. Complement. Alternat. Med. 2014, 495840. doi:10.1155/2014/ 495840

Li, S., Lü, A. P., and Jia, H. W. (2002). Therapeutic actions of the chinese herbal formulae with cold and heat properties and their effects on ultrastructures of synoviocytes in rats of the collagen-induced arthritis. J. Tradit. Chin. Med. 22, 296-302. doi:10.3969/j.issn.0255-2922.2002.04.019

Li, S. (2009). Network systems underlying traditional Chinese medicine syndrome and herb formula. Curr. Bioinf. 4, 188-196. doi:10.2174/157489309789071129

Li, S., Zhang, B., Jiang, D., Wei, Y. Y., and Zhang, N. B. (2010). Herb network construction and co-module analysis for uncovering the combination rule of traditional chinese herbal formulae. BMC Bioinformatics. 11, S6. doi:10.1186/ 1471-2105-11-S11-S6

Li, S., and Zhang, B. (2013). Traditional chinese medicine network pharmacology: theory, methodology and application. Chin. J. Nat. Med. 11, 110-120. doi:10. 1016/S1875-5364(13)60037-0

Li, S., Zhang, Z. Q., Wu, L. J., Zhang, X. G., Li, Y. D., and Wang, Y. Y. (2007). Understanding ZHENG in traditional Chinese medicine in the context of neuro-endocrine-immune network. IET Syst. Biol. 1, 51-60. doi:10.1049/ietsyb:20060032

Li, Y., Hong, Y., Han, Y., Wang, Y., and Xia, L. (2016a). Chemical characterization and antioxidant activities comparison in fresh, dried, stir-frying and carbonized ginger. J. Chromatogr. B. Analyt. Technol. Biomed. Life. Sci. 1011, 223-232. doi:10.1016/j.jchromb.2016.01.009

Li, Y., Wang, P., Chai, M. J., Yang, F., Li, H. S., Zhao, J., et al. (2016b). Effects of berberine on serum inflammatory factors and carotid atherosclerotic plaques in patients with acute cerebral ischemic stroke. China J. Chin. Mater. Med. 41, 4066-4071. doi:10.4268/cjcmm20162128

Liang, F., Li, L., Wang, M. L., Niu, X. Y., Zhan, J. P., He, X. J., et al. (2013). Molecular network and chemical fragment-based characteristics of medicinal herbs with cold and hot properties from chinese medicine. J. Ethnopharmacol. 148, 770-779. doi:10.1016/j.jep.2013.04.055

Liang, Y., Xu, X., Yin, M., Zhang, Y., Huang, L., Chen, R., et al. (2019). Effects of berberine on blood glucose in patients with type 2 diabetes mellitus: a systematic literature review and a meta-analysis. Endocr. J. 66, 51-63. doi:10.1507/endocri.EJ18-0109

Limanaqi, F., Biagioni, F., Busceti, C. L., Polzella, M., Fabrizi, C., and Fornai, F. (2020). Potential antidepressant effects of Scutellaria baicalensis, Hericium erinaceus and Rhodiola rosea. Antioxidants. 9, 234. doi:10.3390/antiox9030234

Lin, M. T., Liu, G. G., Wu, W. L., and Chern, Y. F. (1980). Effects of Chinese herb, huang chin (Scutellaria baicalensis Georgi) on thermoregulation in rats. Jpn. J. Pharmacol. 30, 59-64. doi:10.1254/jjp.30.59

Liu, J., Li, Y., Yang, P., Wan, J., Chang, Q., Wang, T. T. Y., et al. (2017). Gypenosides reduced the risk of overweight and insulin resistance in C57BL/6J mice through modulating adipose thermogenesis and gut microbiota. J. Agric. Food Chem. 65, 9237-9246. doi:10.1021/acs.jafc.7b03382

Liu, S., Lu, F., Wang, X., Sun, W., Chen, P., and Dong, W. (2011). Metabolomic study of a rat fever model induced with 2,4-dinitrophenol and the therapeutic effects of a crude drug derived from coptis chinensis. Am. J. Chin. Med. 39, 95-109. doi:10.1142/S0192415X11008671

Liu, J., Yue, S., Yang, Z., Feng, W., Meng, X., Wang, A., et al. (2018). Oral hydroxysafflor yellow A reduces obesity in mice by modulating the gut microbiota and serum metabolism. Pharmacol. Res. 134, 40-50. doi:10.1016/ j.phrs.2018.05.012

Liu, X., Yang, J., Fu, J., Xie, T. G., Jiang, P. C., Jiang, Z. H., et al. (2018). Phytochemical and chemotaxonomic studies on the twigs of cinnamomum cassia (Lauraceae). Biochem. Systemat. Ecol. 81, 45-48. doi:10.1016/j.bse.2018. 09.004 
Lv, C., Yuan, X., Zeng, H. W., Liu, R. H., and Zhang, W. D. (2017). Protective effect of cinnamaldehyde against glutamate-induced oxidative stress and apoptosis in PC12 cells. Eur. J. Pharmacol. 815, 487-494. doi:10.1016/j.ejphar.2017.09.009

Lyu, H. N., Kwak, H. Y., Lee, D. Y., Kim, K. T., and Baek, N. I. (2008). Isolation of flavonoids from processed Aconiti tuber. J. Appl. Biol. Chem. 51, 165-168. doi:10.3839/jabc.2008.029

Makino, T., Kato, K., and Mizukami, H. (2009). Processed aconite root prevents cold-stress-induced hypothermia and immuno-suppression in mice. Biol. Pharm. Bull. 32, 1741-1748. doi:10.1248/bpb.32.1741

Mamindla, S., Koganti, V., Ravouru, N., and Koganti, B. (2017). Effect of Cinnamomum cassia on the pharmacokinetics and pharmacodynamics of pioglitazone. Curr. Clin. Pharmacol. 12, 41-49. doi:10.2174/ 1574884712666170207152020

Mei, M. F. (2011). A systematic analysis of the theory and practice of syndrome differentiation. Chin. J. Integr. Med. 17, 803-810. doi:10.1007/s11655-0110890-0

Meng, F. C., Wu, Z. F., Yin, Z. Q., Lin, L. G., Wang, R., and Zhang, Q. W. (2018). Coptidis rhizoma and its main bioactive components: recent advances in chemical investigation, quality evaluation and pharmacological activity. Chin. Med. 13, 13. doi:10.1186/s13020-018-0171-3

Min, G. B. (2009). Observation on the curative effect of banxia xiexin decoction in the treatment of functional dyspepsia with mixed cold and hot syndrome. Guangxi J. Tradit. Chin. Med. 5, 30-31

Miyamoto, M., Matsuzaki, K., Katakura, M., Hara, T., Tanabe, Y., and Shido, O. (2015). Oral intake of encapsulated dried ginger root powder hardly affects human thermoregulatory function, but appears to facilitate fat utilization. Int. J. Biometeorol. 59, 1461-1474. doi:10.1007/s00484-015-0957-2

Oh, O. (2003). Zingiber officinale (ginger). Alternative Med. Rev. 8, 331

Ota, M., Nakazaki, J., Tabuchi, Y., Ono, T., and Makino, T. (2019). Historical and pharmacological studies on Rehmannia root processing- trends in usage and comparison of the immunostimulatory effects of its products with or without steam processing and pretreatment with liquor. J. Ethnopharmacol. 242, 112059. doi:10.1016/j.jep.2019.112059

Pandith, S. A., Hussain, A., Bhat, W. W., Dhar, N., Qazi, A. K., Rana, S., et al. (2014). Evaluation of anthraquinones from himalayan rhubarb (Rheum emodi Wall. ex Meissn.) as antiproliferative agents. South Afr. J. Bot. 95, 1-8. doi:10. 1016/j.sajb.2014.07.012

Pandith, S. A., Dar, R. A., Lattoo, S. K., Shah, M. A., and Reshi, Z. A. (2018). Rheum australe, an endangered high-value medicinal herb of North Western Himalayas: a review of its botany, ethnomedical uses, phytochemistry and pharmacology. Phytochemistry Rev. 17, 573-609. doi:10.1007/s11101-018-9551-7

Panthi, S., Jing, X., Gao, C., and Gao, T. (2017). Yang-warming method in the treatment of diabetic peripheral neuropathy: an updated systematic review and meta-analysis. BMC Compl. Alternative Med. 17, 424. doi:10.1186/s12906-0171927-5

Pardede, A., Adfa, M., Kusnanda, A. J., Ninomiya, M., and Koketsu, M. (2017). Flavonoid rutinosides from cinnamomum parthenoxylon leaves and their hepatoprotective and antioxidant activity. Med. Chem. Res. 26, 2074-2079. doi:10.1007/s00044-017-1916-8

Park, H. G., Yoon, S. Y., Ji, Y. C., Lee, G. S., Choi, J. H., Chan, Y. S., et al. (2007). Anticonvulsant effect of wogonin isolated from Scutellaria baicalensis. Eur. J. Pharmacol. 574, 112-119. doi:10.1016/j.ejphar.2007.07.011

Perrins, J. D. (1862). XLIII.-On berberine-contributions to its history and revision of its formula. J. Am. Chem. Soc. 15, 18. doi:10.1039/js8621500339

Porter, D., Cochrane, S., and Zhu, X. (2017). Current usage of traditional chinese medicine for breast cancer-a narrative approach to the experiences of women with breast cancer in australia-a pilot study. Medicines. 4, 20. doi:10.3390/ medicines4020020

Qi, L., Ma, Y., Zhong, F., and Shen, C. (2018). Comprehensive quality assessment for Rhizoma Coptidis based on quantitative and qualitative metabolic profiles using high performance liquid chromatography, Fourier transform nearinfrared and Fourier transform mid-infrared combined with multivariate statistical analysis. J. Pharmaceut. Biomed. Anal. 161, 436-443. doi:10.1016/j. jpba.2018.09.012

Qin, X., Jiang, M., Zhao, Y., Gong, J., Su, H., Yuan, F., et al. (2020). Berberine protects against diabetic kidney disease via promoting PGC-1alpha-regulated mitochondrial energy homeostasis. Br. J. Pharmacol. 177, 3646-3661. doi:10. 1111/bph.14935
Regestein, Q. R. (2012). "Oh, east is east and west is west, and never the twain shall meet"-Rudyard Kipling. Menopause. 12, 1291-1293. doi:10.1097/gme. ob013e3182752f1b

Rokaya, M. B., Munzbergova, Z., Timsina, B., and Bhattarai, K. R. (2012). Rheum australe D. Don: a review of its botany, ethnobotany, phytochemistry and pharmacology. J. Ethnopharmacol. 141, 761-774. doi:10.1016/j.jep.2012.03.048

Rong, R., Li, R. R., Hou, Y. B., Li, J., Ding, J. X., Zhang, C. B., et al. (2016). MahuangXixin-Fuzi Decoction reduces the infection of influenza a virus in kidney-Yang deficiency syndrome mice. J. Ethnopharmacol. 192, 217-224. doi:10.1016/j.jep. 2016.07.017

Sabir, M., and Bhide, N. K. (1971). Study of some pharmacological actions of berberine. Indian J. Physiol. Pharmacol. 15, 111-132

Samad, M. B., Mohsin, M. N. A. B., Razu, B. A., Hossain, M. T., Mahzabeen, S., Unnoor, N., et al. (2017). 6-Gingerol, from Zingiber officinale, potentiates GLP1 mediated glucose-stimulated insulin secretion pathway in pancreatic $\beta$-cells and increases RAB8/RAB10-regulated membrane presentation of GLUT4 transporters in skeletal muscle to improve hyperglycemia in Leprdb/db type 2 diabetic mice. BMC Compl. Alternative Med. 17, 395. doi:10.1186/s12906017-1903-0

Sang, H. S., Lee, S. Y., Ju, S. K., Son, K. H., and Kang, S. S. (2005). Norditerpenoid alkaloids and other components from the processed tubers of aconitum carmichaeli. Arch. Pharm. Res. 28, 1239-1243. doi:10.1007/BF02978206

Schneeberger, M., Everard, A., Gomez-Valades, A. G., Matamoros, S., Ramirez, S., Delzenne, N. M., et al. (2015). Akkermansia muciniphila inversely correlates with the onset of inflammation, altered adipose tissue metabolism and metabolic disorders during obesity in mice. Sci. Rep. 5, 16643. doi:10.1038/ srep16643

Sebaia, H., Rtibi, K., Selmia, S., Jridib, M., Baltia, R., and Marzoukia, L. (2019). Modulating and opposite actions of two aqueous extracts prepared from cinnamomum cassia $\mathrm{L}$. bark and quercus ilex $\mathrm{L}$. on the gastrointestinal tract in rats. RSC $A d v .9,21695-21706$. doi:10.1039/C9RA02429H

Semwal, R. B., Semwal, D. K., Combrinck, S., and Viljoen, A. M. (2015). Gingerols and shogaols: important nutraceutical principles from ginger, Phytochemistry. 117, 554-568. doi:10.1016/j.phytochem.2015.07.012

Shao, F., Wang, D. Q., Xiong, W., Zhang, P. Z., Ma, G. Q., Liu, R. H., et al. (2017). A new pyridine alkaloid from zingiberis rhizoma. Nat. Prod. Res. 31, 1486-1489. doi:10.1080/14786419.2016

Shao, S. Y., and Li, Q. (2019). Research progress in the treatment of colorectal cancer in classical prescriptions. TMR. Cancer. 2, 200-205

Sharma, P. K., Singh, V., Ali, M., and Kumar, S. (2016). Effect of ethanolic extract of zingiber officinale Roscoe on central nervous system activity in mice. Indian J. Exp. Biol. 54, 664-669

Shen, J., Wang, J., Shang, E. X., Tang, Y. P., Kai, J., Cao, Y. J., et al. (2016). The dosage-toxicity-efficacy relationship of kansui and licorice in malignant pleural effusion rats based on factor analysis. J. Ethnopharmacol. 186, 251-256. doi:10. 1016/j.jep.2016.04.012

Shen, Y., Fukushima, M., Ito, Y., Muraki, E., Hosono, T., Seki, T., et al. (2010). Verification of the antidiabetic effects of cinnamon (Cinnamomum zeylanicum) using insulin-uncontrolled type 1 diabetic rats and cultured adipocytes. Biosci. Biotechnol. Biochem. 74, 2418-2425. doi:10.1271/bbb.100453

Shihabudeen, H. M. S., Priscilla, D. H., and Thirumurugan, K. (2011). Cinnamon extract inhibits alpha-glucosidase activity and dampens postprandial glucose excursion in diabetic rats. Nutr. Metab. 8, 46. doi:10.1186/1743-7075-8-46

Shinjyo, N., Waddell, G., and Green, J. (2020). A tale of two cinnamons: a comparative review of the clinical evidence of Cinnamomum verum and $C$. cassia as diabetes interventions. J. Herb. Med. 21, 100342. doi:10.1016/j.hermed. 2020.100342

Song, M. Y., Kang, S. Y., Kang, A., Hwang, J. H., Park, Y. K., and Jung, W. (2017). Cinnamomum cassia prevents high-fat diet-induced obesity in mice through the increase of muscle energy. Am. J. Chin. Med. 45, 1017-1031. doi:10.1142/ S0192415X17500549

Su, S. B., Lu, A., Li, S., and Jia, W. (2012). Evidence-based ZHENG: a traditional Chinese medicine syndrome. Evid. Based. Complement. Alternat. Med. 2012, 246538. doi:10.1155/2012/246538

Sui, F., Huo, H. R., Zhang, C. B., Yang, N., Guo, J. Y., Du, X. L., et al. (2010a). Emodin down-regulates expression of TRPV1 mRNA and its function in DRG neurons in vitro. Am. J. Chin. Med. 38, 789-800. doi:10.1142/ S0192415X1000824X 
Sui, F., Yang, N., Zhang, C., Du, X., Li, L., Weng, X., et al. (2010b). Effects of ingredients from Chinese herbs with nature of cold or hot on expression of TRPV1 and TRPM8. China J. Chin. Mater. Med. 35, 1594-1598. doi:10.4268/ cjcmm 20101220

Sun, L., Liu, L., Zong, S., Wang, Z., Zhou, J., Xu, Z., et al. (2016). Traditional Chinese medicine Guizhi Fuling capsule used for therapy of dysmenorrhea via attenuating uterus contraction. J. Ethnopharmacol. 191, 273-279. doi:10.1016/j. jep.2016.06.042

Sun, L., You, G. J., Cao, X. X., Wang, M., and Ren, X. L. (2019). Comparative investigation for raw and processed Aconiti Lateralis Radix using chemical UPLC-MS profiling and multivariate classification techniques. J. Food Drug Anal. 27, 365-372. doi:10.1016/j.jfda.2018.10.006

Sun, Z. Y., Zhao, Y. L., Wang, J. B., Zhang, L., Wei, S. S., Jiang, F. J., et al. (2012). Research on Fuzi based on animal termotropism behavior to discover if it has fewer "hot" characteristics without Ganjiang. J. Tradit. Chin. Med. 32, 208-214. doi:10.1016/S0254-6272(13)60013-8

Tamura, Y., Iwasaki, Y., Narukawa, M., and Watanabe, T. (2012). Ingestion of cinnamaldehyde, a TRPA1 agonist, reduces visceral fats in mice fed a high-fat and high-sucrose diet. J. Nutr. Sci. Vitaminol. 58, 9-13. doi:10.3177/jnsv.58.9

Tan, A., Huang, H. Y., Zhang, P., and Li, S. (2019). Network-based cancer precision medicine: a new emerging paradigm. Canc. Lett. 458, 39-45. doi:10.1016/j. canlet.2019.05.015

Tan, H. L., Chan, K. G., Pusparajah, P., Duangjai, A., Saokaew, S., Mehmood Khan, T., et al. (2016). Rhizoma Coptidis: a potential cardiovascular protective agent. Front. Pharmacol. 7, 362. doi:10.3389/fphar.2016.00362

Tan, Y., Liu, X. R., Lu, C., He, X. J., Li, J., Xiao, C., et al. (2014). Metabolic profiling reveals therapeutic biomarkers of processed Aconitum carmichaeli Debx in treating hydrocortisone induced kidney-Yang deficiency syndrome rats. J. Ethnopharmacol. 152, 585-593. doi:10.1016/j.jep.2014.02.011

Tang, L., Gong, Y., Lv, C., Ye, L., Liu, L., and Liu, Z. Q. (2012). Pharmacokinetics of aconitine as the targeted marker of Fuzi (Aconitum carmichaeli) following single and multiple oral administrations of Fuzi extracts in rat by UPLC/MS/ MS. J. Ethnopharmacol. 141, 736-741. doi:10.1016/j.jep.2011.08.070

Teodoro, J. S., Zouhar, P., Flachs, P., Bardova, K., Janovska, P., Gomes, A. P., et al. (2014). Enhancement of brown fat thermogenesis using chenodeoxycholic acid in mice. Int. J. Obes. 38, 1027-1034. doi:10.1038/ijo.2013.230

Tsai, C. C., Lin, M. T., Wang, J. J., Liao, J. F., and Huang, W. T. (2006). The antipyretic effects of baicalin in lipopolysaccharide-evoked fever in rabbits. Neuropharmacology. 51, 709-717. doi:10.1016/j.neuropharm.2006.05.010

Ung, C. Y., Li, H., Cao, Z. W., Li, Y. X., and Chen, Y. Z. (2007). Are herb-pairs of traditional Chinese medicine distinguishable from others? Pattern analysis and artificial intelligence classification study of traditionally defined herbal properties. J. Ethnopharmacol. 111, 371-377. doi:10.1016/ j.jep.2006.11.037

Vishwakarma, S. L., Pal, S. C., Kasture, V. S., and Kasture, S. B. (2002). Anxiolytic and antiemetic activity of zingiber officinale. Phytother Res. 16, 621-626. doi:10. 1002/ptr.948

Wan, H. Y., Kong, X. Y., Li, X. M., Zhu, H. W., Su, X. H., and Lin, N. (2014). Effect of traditional Chinese medicines with different properties on thermoregulation and temperature-sensitive transient receptor potentialion channel protein of rats with yeast-induced fever. China J. Chin. Mater. Med. 39, 3813-3818. doi:10. 4268/cjcmm20141930

Wang, C., Wu, X., Chen, M., Duan, W., Sun, L., Yan, M., et al. (2007). Emodin induces apoptosis through caspase 3-dependent pathway in HK-2 cells. Toxicology. 231, 120-128. doi:10.1016/j.tox.2006.11.064

Wang, H., Mu, W., Shang, H., Lin, J., and Lei, X. (2014). The antihyperglycemic effects of Rhizoma Coptidis and mechanism of actions: a review of systematic reviews and pharmacological research. BioMed Res. Int. 2014, 798093. doi:10. 1016/j.fitote.2019.03.003

Wang, J., Zhang, L., Dong, L., Hu, X., Feng, F., and Chen, F. (2019). 6-Gingerol, a functional polyphenol of ginger, promotes browning through an AMPKdependent pathway in 3T3-L1 adipocytes. J. Agric. Food Chem. 67, 14056-14065. doi:10.1021/acs.jafc.9b05072

Wang, D., Liu, C. D., Li, H. F., Tian, M. L., Pan, J. Q., Shu, G., et al. (2020). LSD1 mediates microbial metabolite butyrate-induced thermogenesis in brown and white adipose tissue. Metabolism. 102, 154011. doi:10.1016/j.metabol.2019.154011

Wang, J., Su, B. Z., Jiang, H. Q., Cui, N., Yu, Z. Y., Yang, Y. H., et al. (2020). Traditional uses, phytochemistry and pharmacological activities of the genus cinnamomum (Lauraceae): a review. Fitoterapia. 146, 104675. doi:10.1016/j. fitote.2020.104675

Wang, X., Wu, M., Lai, X. X., Zheng, J. H., Hu, M. H., Li, Y., et al. (2020). Network pharmacology to uncover the biological basis of spleen qi deficiency syndrome and herbal treatment. Oxid. Med. Cell. Longev. 2020, 2974268. doi:10.1155/ 2020/2974268

Wang, K., Feng, X., Chai, L., Cao, S., and Qiu, F. (2017). The metabolism of berberine and its contribution to the pharmacological effects. Drug Metab. Rev. 49, 139-157. doi:10.1080/03602532.2017.1306544

Wang, M., Chen, L., Liu, D., Chen, H., Tang, D. D., and Zhao, Y. Y. (2017). Metabolomics highlights pharmacological bioactivity and biochemical mechanism of traditional chinese medicine. Chem. Biol. Interact. 273, 133-141. doi:10.1016/j.cbi.2017.06.011Wang

Wang, Q. H., Yang, X., Li, X. L., Yang, B. Y., Wang, Y. H., and Kuang, X. H. (2015). Based on proteomics to study the property and flavor of radix aconiti lateralis preparata, rhizoma zingiberis, pricklyash peel. World. J. Tradit. Chin. Med. 10, 1824-1836.

Wang, S. P., Hu, Y. Y., Tan, W., Wu, X., Chen, R., Cao, J. L., et al. (2012). Compatibility art of traditional chinese medicine: from the perspective of herb pairs. J. Ethnopharmacol. 143, 412-423. doi:10.1016/j.jep.2012.07.033

Wang, X. Y., Li, F., and Xue, F. Z. (2012). Study on appropriate statistical pattern recognition model for relationship between cold-hot natures and polysaccharides based on HPLC of 60 traditional chinese medicines. Chin. J. Tradit. Chin. Med. Pharm. 4, 943-947

Wang, S., Tian, M., Yang, R. L., Jing, Y. T., Chen, W., Wang, J. L., et al. (2018). 6Gingerol ameliorates behavioral changes and atherosclerotic lesions in ApoE ${ }^{-}$mice exposed to chronic mild stress. Cardiovasc. Toxicol. 18, 420-430. doi:10. 1007/s12012-018-9452-4

Wang, Y., Zhang, Y., Shi, Y. Q., Pan, X. H., and Cao, P. (2018). Antibacterial effects of cinnamon (Cinnamomum zeylanicum) bark essential oil on porphyromonas gingivalis. Microb. Pathog. 116, 26-32. doi:10.1016/j.micpath.2018.01.009

Wang, Z. L., Wang, S., Kuang, Y., Hu, Z. M., Qiao, X., and Ye, M. (2018). A comprehensive review on phytochemistry, pharmacology, and flavonoid biosynthesis of Scutellaria baicalensis. Pharm. Biol. 56, 465-484. doi:10. 1080/13880209.2018.1492620

Wang, W. X. (2016). Analysis of characteristics of chemical pharmacological action and clinical application of ginger. Clin. Res. Tradit. Chin. Med. 8, 146-148. doi:10.3969/j.issn.1674-7860.2016.06.073

Wang, Y., Zhou, S. J., Wang, M., Liu, S. Y., Hu, Y. J., He, C. W., et al. (2016). UHPLC/Q-TOFMS-based metabolomics for the characterization of cold and hot properties of Chinese materia medica. J. Ethnopharmacol. 179, 234-242. doi:10.1016/j.jep.2015.12.061

Wang, Y. H., Avula, B., Nanayakkara, N. P., Zhao, J., and Khan, I. A. (2013). Cassia cinnamon as a source of coumarin in cinnamon-flavored food and food supplements in the United States. J. Agric. Food Chem. 61, 4470-4476. doi:10.1021/jf4005862

Wei, G. H., Fu, X. J., and Wang, Z. G. (2019). Multisolvent similarity measure of Chinese herbal medicine ingredients for cold-hot nature identification. J. Chem. Inf. Model. 59, 5065-5073. doi:10.1021/acs.jcim.9b00682

Wen, J., Zou, W., Wang, R., Liu, H., Yang, Y., Li, H., et al. (2019). Cardioprotective effects of Aconiti Lateralis Radix Praeparata combined with Zingiberis Rhizoma on doxorubicin-induced chronic heart failure in rats and potential mechanisms. J. Ethnopharmacol. 238, 111880. doi:10.1016/j.jep.2019.111880

Worthmann, A., John, C., Ruhlemann, M. C., Baguhl, M., Heinsen, F. A., Schaltenberg, N., et al. (2017). Cold-induced conversion of cholesterol to bile acids in mice shapes the gut microbiome and promotes adaptive thermogenesis. Nat. Med. 23, 839-849. doi:10.1038/nm.4357

Xia, F. B., Liu, C. H., and Wan, J. B. (2020). Characterization of the cold and hot natures of raw and processed Rehmanniae Radix by integrated metabolomics and network pharmacology. Phytomedicine. 74, 153071. doi:10.1016/j.phymed. 2019.153071

Xiao, S., Zhang, Z., Chen, M., Zou, J., Jiang, S., Qian, D., et al. (2019). Xiexin Tang ameliorates dyslipidemia in high-fat diet-induced obese rats via elevating gut microbiota-derived short chain fatty acids production and adjusting energy metabolism. J. Ethnopharmacol. 241, 112032. doi:10.1016/ j.jep.2019.112032

Xie, H. S. (2017). Historical review of the origins of the nine different schools of traditional chinese medicine. Am. J. Chin. Med. 12, 59-75 
Yan, S. G., Zhou, Y. X., Yi, H., and Huang, Y. X. (2012). Experiment study of treatment effects and subdivisions of Wumei Pill on TNBS-induced ulcerative colitis. Chin. J. Tradit. Chin. Med. Pharm. 4, 890-895

Yang, X., Wu, Y., Zhang, C., Fu, S., Zhang, J., and Fu, C. (2020). Extraction, structural characterization, and immunoregulatory effect of a polysaccharide fraction from radix aconiti lateralis preparata (Fuzi). Int. J. Biol. Macromol. 143, 314-324. doi:10.1016/j.ijbiomac.2019.11.208

Yao, J., Kong, W., and Jiang, J. (2015). Learning from berberine: treating chronic diseases through multiple targets. Sci. China Life Sci. 58, 854-859. doi:10.1007/ s11427-013-4568-z

Yi, J., Ye, X., Wang, D., He, K., Yang, Y., Liu, X., et al. (2013). Safety evaluation of main alkaloids from Rhizoma Coptidis. J. Ethnopharmacol. 145, 303-310. doi:10.1016/j.jep.2012.10.062

Yu, B., Cao, Y., and Xiong, Y. K. (2015) Pharmacokinetics of aconitine-type alkaloids after oral administration of Fuzi (Aconiti Lateralis Radix Praeparata) in rats with chronic heart failure by microdialysis and ultra-high-performance liquid chromatography-tandem mass spectrometry. J. Ethnopharmacol. 165, 173-179. doi:10.1016/j.jep.2015.01.057

Yu, S. Y., Li, C., Ding, Y. S., Huang, S., Wang, W., Wu, Y. Y., et al. (2020). Exploring the 'cold/hot' properties of traditional Chinese medicine by cell temperature measurement. Pharm. Biol. 58, 208-218. doi:10.1080/13880209.2020.1732429

Yu, Y. H., Wang, S. J., Teng, J. L., Ji, X. M., Wu, Z. C., Ma, Q. C., et al. (2012). Effects of Radix Aconiti Lateralis Preparata and Rhizoma Zingiberis on energy metabolism and expression of the genes related to metabolism in rats. Chin. J. Integr. Med. 18, 23-29. doi:10.1007/s11655-012-0964-7

Zargar, B. A., Masoodi, M. H., Ahmed, B., and Ganie, S. A. (2011). Phytoconstituents and therapeutic uses of rheum emodi wall. Ex Meissn. Food. Chem. 128, 585-589. doi:10.1016/j.foodchem.2011.03.083

Zhang, B., Wang, X., and Li, S. (2013). An integrative platform of TCM network pharmacology and its application on a herbal formula, qing-luo-yin. Evid. Based. Complement. Alternat. Med. 2013, 456747. doi:10.1155/2013/456747

Zhang, C., Fan, L., Fan, S., Wang, J., and Yu, L. (2019). Cinnamomum cassia Presl: a review of its traditional uses, phytochemistry, pharmacology and toxicology. Molecules. 24, 3473. doi:10.3390/molecules24193473

Zhang, H. X., Chi, X. S., Pan, W. C., Wang, S. J., Zhang, Z., Zhao, H. J., et al. (2020). Antidepressant mechanism of classical herbal formula lily bulb and Rehmannia decoction: insights from gene expression profile of medial prefrontal cortex of mice with stress-induced depression-like behavior. Gene Brain Behav. 19, e12649. doi:10.1111/gbb.12649

Zhang, J. H., Zhu, Y., Fan, X. H., and Zhang, B. L. (2015). Efficacy-oriented compatibility for component-based chinese medicine. Acta Pharmacol. Sin. 36, 654-658. doi:10.1038/aps.2015.8

Zhang, R. Z., Qiu, H., Wang, N., Long, F. L., and Mao, D. W. (2015). Effect of Rheum palmatum L. on NF- $\kappa \mathrm{B}$ signaling pathway of mice with acute liver failure. Asian. Pac. J. Trop. Med. 8, 841-847. doi:10.1016/j.apjtm.2015.09.011

Zhang, Q., Chen, X., Luo, Y. J., Ren, H. P., and Qiao, T. K. (2017). Fuzi enhances anti-tumor efficacy of radiotherapy on lung cancer. J. Cancer. 8, 3945-3951. doi:10.7150/jca.22162.eCollection2017

Zhang, S. Y. (2011). The TCM etiology, pathogenesy and differential treatment for sjogren's syndrome. J. Tradit. Chin. Med. 31, 73-78. doi:10.1016/s02546272(11)60017-4

Zhang, X. Y., Sukhchuluun, G., Bo, T. B., Chi, Q. S., Yang, J. J., Chen, B., et al. (2018). Huddling remodels gut microbiota to reduce energy requirements in a small mammal species during cold exposure. Microbiome. 6, 103. doi:10.1186/ s40168-018-0473-9

Zhang, Q., Yang, H., An, J., Zhang, R., Chen, B., and Hao, D. J. (2016). Therapeutic effects of traditional Chinese medicine on spinal cord injury: a promising supplementary treatment in future. Evid. Based. Complement. Alternat. Med. 2016, 8958721. doi:10.1155/2016/8958721

Zhang, Y. Q., Mao, X., Guo, Q. Y., Lin, N., and Li, S. (2016). Network pharmacology-based approaches capture essence of chinese herbal medicines. Chin. Herb. Med. 8, 107-116. doi:10.1016/S1674-6384(16)60018-7

Zhang, Y. X., and Feng, J., (1987). Lessons from misuse of cold and hot traditional Chinese medicine. Tradit. Chin. Med. Res. 1, 29-30

Zhao, D., Wang, J., Cui, Y., and Wu, X. (2012). Pharmacological effects of Chinese herb aconite (Fuzi) on cardiovascular system. J. Tradit. Chin. Med. 32, 308-313. doi:10.1016/s0254-6272(13)60030-8
Zhao, H. Y., Zhang, F., and Fan, S. D. (2002). Effects of baicalin on contents of PGE2 and cAMP in hypothalamus of fever rats. Zhongguo Ying Yong Sheng $L i$ Xue Za Zhi. 18, 139-141

Zhao, L. H., Sun, Z. H., Yang, L. M., Cui, R. J., Yang, W., and Li, B. J. (2020). Neuropharmacological effects of aconiti lateralis radix praeparata. Chin. Exp. Pharmacol. Physiol. 47, 531-542. doi:10.1111/1440-1681.13228

Zhao, L., Zhang, S., Wang, Z., Wang, C., Huang, S., Shen, H., et al. (2013a). Efficacy of modified Banxia Xiexin Decoction on functional dyspepsia of cold and heat in complexity syndrome: a randomized controlled trial. Evid. Based. Complement. Alternat. Med. 2013, 812143. doi:10.1155/2013/812143

Zhao, L., Wu, H., Qiu, M., Sun, W., Wei, R., Zheng, X., et al. (2013b). Metabolic signatures of kidney Yang deficiency syndrome and protective effects of two herbal extracts in rats using GC/TOF MS. Evid. Based. Complement. Alternat. Med. 2013, 540957. doi:10.1155/2013/540957

Zhao, T., Tang, H., Xie, L., Zheng, Y., Ma, Z., Sun, Q., et al. (2019). Scutellaria baicalensis Georgi. (Lamiaceae): a review of its traditional uses, botany, phytochemistry, pharmacology and toxicology. J. Pharm. Pharmacol. 71, 1353-1369. doi:10.1111/jphp.13129

Zhao, Y. J., and Song, Q. Z. (2011). Treatment of 48 cases of functional dyspepsia with mixed cold and heat by Banxia Xiexin Decoction. Shaanxi J. Tradit. Chin. Med. 9, 1144-1146

Zhao, Y. L., Wang, J. B., Xiao, X. H., Zhao, H. P., Zhou, C. P., Zhang, X. R., et al. (2011). Study on the cold and hot properties of medicinal herbs by thermotropism in mice behavior. J. Ethnopharmacol. 133, 980-985. doi:10. 1016/j.jep.2010.09.014

Zheng, J. H., Wu, M., Wang, H. Y., Li, S. S., Wang, X., Li, Y., et al. (2018). Network pharmacology to unveil the biological basis of healthstrengthening herbal medicine in cancer treatment. Cancers. 10, 461. doi:10.3390/cancers10110461

Zheng, Q., Zhao, Y., Wang, J., Liu, T., Zhang, B., Gong, M., et al. (2014). Spectrumeffect relationships between UPLC fingerprints and bioactivities of crude secondary roots of Aconitum carmichaelii Debeaux (Fuzi) and its three processed products on mitochondrial growth coupled with canonical correlation analysis. J. Ethnopharmacol. 153, 615-623. doi:10.1016/j.jep.2014.03.01

Zheng, W. J., Wang, G. F., Zhang, Z., Wang, Z. G., and Ma, K. (2019). Research progress on classical traditional Chinese medicine formula Liuwei Dihuang pills in the treatment of type 2 diabetes. Biomed. Pharmacother. 121, 109564. doi:10. 1016/j.biopha.2019.109564

Zhou, B. C., Li, T. Y. N., Yang, M. N., Pang, J. X., Min, L. Y., and Han, J. X. (2019). Characterization of the hot and cold medicinal properties of traditional Chinese herbs by spontaneous photon emission ratio of mice. J. Ethnopharmacol. 243, 112108. doi:10.1016/j.jep.2019.112108

Zhou, N., Yang, Y. Y., Li, K., Ke, Y. Y., Zheng, X. K., Feng, W. S., et al. (2019). Integrating strategies of chemistry, biochemistry and metabolomics for characterization of the medication principle of "treating cold/heat syndrome with hot/cold herbs. J. Ethnopharmacol. 239, 111899. doi:10.1016/j.jep.2019.111899

Zhou, G., Tang, L., Zhou, X., Wang, T., Kou, Z., and Wang, Z. (2015). A review on phytochemistry and pharmacological activities of the processed lateral root of Aconitum carmichaelii Debeaux. J. Ethnopharmacol. 160, 173-193. doi:10.1016/j.jep. 2014.11.043

Zhou, S. J., Meng, J., and Liu, B. (2017). Investigation into the pharmacokinetic-pharmacodynamic model of zingiberis rhizoma/zingiberis rhizoma carbonisata and contribution to their therapeutic material basis using artificial neural networks. RSC Adv. 7, 25488-25496. doi:10.1039/C7RA01478C

Zhou, Y., Guo, Z. J., Han, L., Li, Y., and Wang, X. M. (2014). Optimization of chloroplast microsatellite PCR conditions and primer screening for endangered Rheum officinale, rheum palmatum, and rheum tanguticum. Genet. Mol. Res. 13, 5787-5794. doi:10.4238/2014.July.29.6

Zhu, R., Liu, H., Liu, C., Wang, L., Ma, R., Chen, B., et al. (2017). Cinnamaldehyde in diabetes: a review of pharmacology, pharmacokinetics and safety. Pharmacol. Res. 122, 78-89. doi:10.1016/j.phrs.2017.05.019

Ziętak, M., Kovatcheva-Datchary, P., Markiewicz, L. H., Ståhlman, M., Kozak, L. P., and Bäckhed, F. (2016). Altered microbiota contributes to reduced diet-induced obesity upon cold exposure. Cell Metabol. 23, 1216-1223. doi:10.1016/j.cmet.2016. 05.001

Zietak, M., and Kozak, L. P. (2016). Bile acids induce uncoupling protein 1dependent thermogenesis and stimulate energy expenditure at 
thermoneutrality in mice. Am. J. Physiol. Endocrinol. Metab. 310, E346-E354. doi:10.1152/ajpendo.00485.2015

Zou, K., Li, Z., Zhang, Y., Zhang, H. Y., Li, B., Zhu, W. L., et al. (2017). Advances in the study of berberine and its derivatives: a focus on anti-inflammatory and anti-tumor effects in the digestive system. Acta Pharmacol. Sin. 38, 157-167. doi:10.1038/aps.2016.125

Zu, Y. X., Lu, H. Y., Liu, W. W., Jiang, X. W., Huang, Y., Li, X., et al. (2019). Jiang Gui Fang activated interscapular brown adipose tissue and induced epididymal white adipose tissue browning through the PPAR $\gamma /$ SIRT1PGC1 $\alpha$ pathway. J. Ethnopharmacol. 248, 112271. doi:10.1016/j.jep.2019. 112271

Zuo, T., Fan, X. S., Tian, S., Jiang, C. X., and Chen, F. (2015). Clinical study on aconite prescriptions with incompatible herbs in different areas based on association rules and analysis on compatibility features. China J. Chin. Mater. Med. 40, 1198-1202. doi:10.4268/cjcmm20150639

Conflict of Interest: The authors declare that the research was conducted in the absence of any commercial or financial relationships that could be construed as a potential conflict of interest.

Copyright (0) 2021 Liu, Feng and Peng. This is an open-access article distributed under the terms of the Creative Commons Attribution License (CC BY). The use, distribution or reproduction in other forums is permitted, provided the original author(s) and the copyright owner(s) are credited and that the original publication in this journal is cited, in accordance with accepted academic practice. No use, distribution or reproduction is permitted which does not comply with these terms. 